\title{
Numerical Simulation of a Gyro-BWO with a Helically Corrugated Interaction Region, Cusp Electron Gun and Depressed Collector
}

\author{
Wenlong He, Craig R. Donaldson, Liang Zhang, Kevin Ronald, \\ Alan D. R. Phelps and Adrian W. Cross \\ SUPA, Department of Physics, University of Strathclyde, Glasgow, G4 ONG \\ Scotland, UK
}

\section{Introduction}

The gyrotron backward wave oscillator (gyro-BWO) is an efficient source of frequency-tunable high-power coherent radiation in the microwave to the terahertz range. It has attracted significant research interest recently due to its potential applications in many areas such as remote sensing, medical imaging, plasma heating and spectroscopy. A gyro-BWO using a helically corrugated interaction region (HCIR) has achieved an even wider frequency tuning range and higher efficiency compared with a conventional gyro-BWO with a smooth-bore cavity. This is due to the existence of an "ideal"eigenwave in the HCIR with a large and constant group velocity when the axial wave number is small.

The eigenwave has a $\mathrm{TE}_{21}$-like cross-sectional electric field distribution. For such a field structure it is favourable to use the second harmonic of the electron cyclotron frequency of an axis-encircling electron beam to interact with the wave. The advantage being that it lowers the required magnetic field strength by a factor of two whilst avoiding undesired parasitic oscillations. Therefore a cusp gun was used to produce an annular, axis-encircling electron beam with high velocity ratio, $\alpha$ (ratio of transverse velocity to axial velocity) for the gyro-BWO. This has inherent advantages over a solid beam for energy recovery due to the reduced beam power density in the collector surface making high power $(\sim \mathrm{kWs})$ continuous wave $(\mathrm{CW})$ operation of a gyro-BWO more feasible. The overall efficiency of the gyro-BWO is further improved by using a four-stage depressed collector which recovers the energy from the spent electrons of the gyro-BWO.

The 3D particle-in-cell (PiC) code MAGIC was used to simulate the electron beam trajectories, beam-wave interaction and wave growth in the gyro-BWO. The trajectories of the electrons were simulated including their emission from the cathode, acceleration in the cusp gun region, transportation and interaction in the helical interaction region and deceleration in the depressed collector. Through the simulations a thermionic cusp electron gun was optimized to produce a $40 \mathrm{keV}, 1.5 \mathrm{~A}$, large-orbit, electron beam with an axial velocity spread $\Delta v_{z} / v_{z}$ of $\sim 8 \%$ and a relative $\alpha$ spread $\Delta \alpha / \alpha$ of $\sim 10 \%$ at an $\alpha$ value of 1.65 . When driven by such a beam the gyro-BWO was simulated to have a $3 \mathrm{~dB}$ frequency bandwidth of $84-104 \mathrm{GHz}$, output power of $10 \mathrm{~kW}$ with an electronic efficiency of $17 \%$. The optimization of the shape 
and dimensions of each stage of the depressed collector using a genetic algorithm achieved an overall recovery efficiency of about $70 \%$, with a minimized back-streaming rate of $4.9 \%$ and maximum heat density on the electrodes of $240 \mathrm{~W} / \mathrm{cm}^{2}$. An overall efficiency of $40 \%$ was therefore simulated for the gyro-BWO.

A number of gyro-BWOs have been investigated both in theory and experiments. Two such experiments at the Naval Research Laboratory (Park et al., 1990) and the National Tsing Hua University (Kou et al., 1993) operating at the fundamental cyclotron harmonic and the fundamental mode of a smooth cylindrical waveguide demonstrated impressive voltage and frequency tuning up to $5 \%$ and $13 \%$, respectively with a very high efficiency of nearly $20 \%$ at power levels of up to $100 \mathrm{~kW}$ at Ka-band frequencies. High-power, high-frequency, coherent radiation sources, especially in the range of $\mathrm{mm}$ and sub-mm wavelengths, have attracted significant research interest recently due to their desirable applications in many areas such as remote sensing (Manheimer et al., 1994), medical imaging (Arnone et al., 1999), plasma heating (Imai et al., 2001) and spectroscopy (Smirnova et al., 1995). Gyro-devices are promising candidates to fulfill such a demand due to the advantages of their characteristic fast wave interaction.

A HCIR has been demonstrated with a wave dispersion that has a near constant group velocity in the region of small axial wavenumber (Burt et al., 2005; 2004; McStravick et al., 2010; Samsonov et al., 2004). This allows broadband microwave amplification to be achieved in a gyrotron traveling wave amplifier (gyro-TWA) and wide frequency tuning in a gyro-BWO without compromising interaction efficiency and output power when compared with its counterparts using cylindrical smooth-bore waveguides (Bratman et al., 2007; 2000). Previous experiments using such a microwave system at Ka-band achieved an output power of $\sim 1 \mathrm{MW}$, an efficiency of $10 \%$, a frequency tuning band of $15 \%$ using a $20 \mathrm{~ns}, 300 \mathrm{keV}$ electron beam (Bratman et al., 2001). Recently a relative frequency-tuning band of $17 \%$ at X-band with $16.5 \%$ electronic efficiency was achieved (Denisov et al., 1998; He et al., 2005) at the second harmonic of the electron cyclotron mode using a three-fold HCIR and an axis-encircling electron beam. Research projects involving a W-band gyro-BWO using a HCIR are in progress at the University of Strathclyde. The setup of the device is shown in Fig. 1.

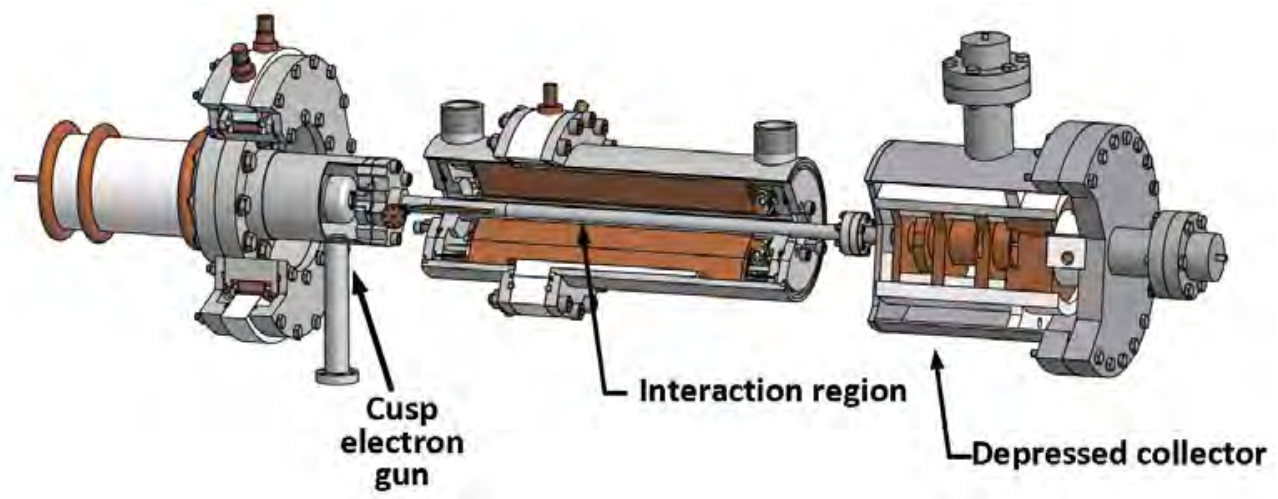

Fig. 1. The experimental setup of the W-band gyro-BWO.

Presented in this chapter is the simulation and optimization of the W-band gyro-BWO by using MAGIC (Goplen et al., 1995; Ludeking et al., 2003), (MAGnetic Insulation Code) by 
Mission Research Laboratory. MAGIC simulates the interaction between charged particles and electromagnetic fields as they evolve in time and space from their initial states. Time and three-dimensional space are divided into finite grids. For each time step, the electromagnetic fields in the three-dimensional grids are solved from the Maxwell equations which are discretized with centered difference approximations. Then the complete Lorentz force equation was used to advance the momenta and coordinates of all charged particles in the simulation under the solved electromagnetic fields. The continuity equation is solved to map charge and current densities onto the grid, which are then used as sources for Maxwell's equations on the next time step. Self consistently solving Maxwell equations, the Lorentz equation and the continuity equation provided a basis for simulating beam field interaction problems.

The simulation and optimization of a thermionic cusp electron gun which generates an annular, axis-encircling electron beam is discussed in section 2 . The simulation of the beam-wave interaction in the HCIR is presented in section 3 . The simulation and optimization of an energy recovery system through a 4-stage depressed collector is given in section 4 . Although it is possible that the integral system of the gyro-BWO, including the electron emissions from the thermionic cathode, beam acceleration in the cusp gun region, propagation in the beam-wave interaction region and deceleration in the depressed collector region and the beam-wave interaction itself can be simulated in one run, the time required to run the whole simulation would be too long. However the total simulation time can be reduced significantly by dividing it into three separate simulations as the system requires different coordinate resolution at different stages.

\section{Simulation of the cusp electron gun}

\subsection{Introduction}

The electron gun choice, design and quality of the transported beam is a very important aspect of any gyro-device. Designing the ideal diode is a complicated process taking into account many different factors including: space-charge forces, the magnetostatic and electrostatic fields and electron emission process. This section discusses the design of a cusp electron gun with numerical and analytical analysis of the cathode and electron beam.

\subsection{Electron guns}

There are a number of electron gun types but in gyro-devices there are three which are most common; the Magnetron Injection Gun (MIG), Pierce-like gun with "kicker"and the cusp electron gun. The MIG gun produces an annular electron beam where the electrons have small orbits each having its own axis, shown in Fig. 2(a). This type of gun is ideal for gyrotrons operating at the fundamental waveguide mode but operation with a harmonic mode is prone to parasitic oscillations. Many high-frequency gyro-devices operate at harmonics (Cooke et al., 1996; Idehara et al., 2004; Wang et al., 2000; 1994) to allow for the use of a larger cavity diameter and to decrease magnetic field strength by a factor of $s$, the harmonic number. An axis-encircling electron beam is ideal for harmonic gyro-devices due to its good mode selectivity as the beam-wave coupling requires that the azimuthal index of the waveguide mode, $m$ to be equal to $s$ (Chu, 1978). There are two such electron guns that can generate this type of beam, the Pierce-like gun with a "kicker"and the cusp electron gun. The Pierce-like gun with a "kicker"produces a solid pencil beam which travels through a magnetic 
"kicker"that induces azimuthal rotation so the beam will travel in a helical path through the interaction region as illustrated in Fig. 2(b). The disadvantages of this electron gun is that operation in the CW mode is difficult and the spent solid beam would cause a "bright spot"on the collector surface and hence damage the system. The cusp electron gun can generate an axis-encircling annular electron beam (see Fig. 2(c)), through a mechanism of beam generation which supports CW operation and allows the $\alpha$ of the beam to be controllable by changing the magnetic field strength at the cathode.

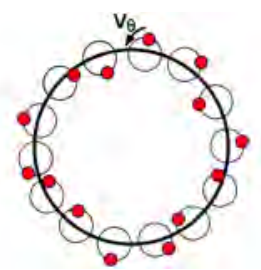

(a) MIG gun beam

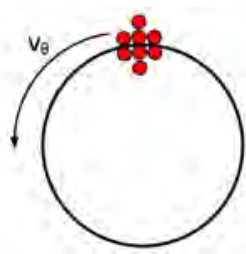

(b) Pierce-like gun beam with a "kicker"

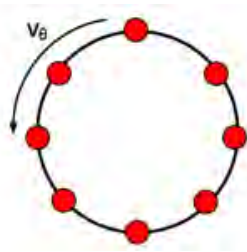

(c) Cusp gun produced beam

Fig. 2. Electron beam profile of various electron gun types.

\subsection{Principle of the cusp electron gun}

The cusp electron gun operates by utilizing two solenoids, one at the cavity region the other, with an opposite direction, just behind the cathode. The combination of magnetic fields results in a cusped magnetic field region in front of the cathode. When the electron beam passes through the cusp, the canonical angular momentum, described in Eq. 1, must be conserved. However, the vector potential $A_{\theta}$ is related to the amplitude of the magnetic field so the equation would then become unbalanced. In order to conserve the momentum, $v_{\theta}$ must change and so the electron beam will rotate around the axis of symmetry.

$$
P_{\theta}=m v_{\theta} r+q r A_{\theta}
$$

where $r$ is the radius of the electrons, $m$ is the electron mass, $q$ is electron charge, $A_{\theta}$ is the vector potential.

It is possible to show (He et al., 2008) that the value of $\alpha$ can be described approximately through Eq. 2.

$$
\alpha=\frac{v_{\perp}}{v_{z}}=\sqrt{\frac{r_{c}^{2}\left|\omega_{c}\right| \omega_{0}}{V_{0}^{2}-r_{c}^{2}\left|\omega_{c}\right| \omega_{0}}}
$$

where $V_{0}$ is the total electron velocity, $\omega=e B / \gamma m_{e}$, subscript " $\mathrm{c}$ " and " 0 " denote the cathode and the downstream uniform magnetic region. $\gamma$ is the Lorentz factor of the electrons at the downstream region, and $e$ and $m_{e}$ are the charge and rest mass of the electron respectively. The radius of the electrons in the cavity magnetic field region can be calculated by using Eq. 3 (Chen, 1974).

$$
r_{0}=\frac{r_{c}}{\sqrt{B_{0} /\left|B_{c}\right|}}
$$




\subsection{Previous research on cusp electron guns}

Initially, transport of an electron beam through opposing magnetic fields (so called "magnetic cusp") was investigated in the 1960's (Schmidt, 1962; Sinnis \& Schmidt, 1963) for plasma confinement applications. Schmidt described a threshold for magnetic mirroring of an electron stream and the effect on the electron trajectory passing through the cusp region. The main conclusion of this paper, with respect to microwave devices, is that the electrons gain azimuthal velocity around the axis of symmetry due to conservation of canonical angular momentum. This theoretical prediction was proven through experimental measurement (Sinnis \& Schmidt, 1963). Building on the work of Schmidt et al., continuous efforts and progress have been made through both theoretical analysis and experimental study in the generation of the cusp-based electron beam sources (Destler \& Rhee, 1977; Rhee \& Destler, 1974). Special attention was paid to methods which can produce an ideal sharp cusp shape by using complex arrays of magnetic coils, magnetic poles and possibly magnetic material inside the cathode (Jeon et al., 2002; Nguyen et al., 1992; Scheitrum et al., 1989; Scheitrum \& True, 1981). This culminated in a "state-of-the-art" cusp gun in 2000 by Northrop Grumman (Gallagher et al., 2000) which generated an electron beam of energy $70 \mathrm{kV}$, current $3.5 \mathrm{~A}$ and velocity ratio 1.5 with a small axial velocity spread of $5 \%$ at a magnetic field of $\sim 0.25 \mathrm{~T}$. Recently gyro-devices have begun to adopt cusp guns as their electron beam sources notably in lower frequency harmonic gyro-devices (McDermott et al., 1996).

A cold cathode cusp gun was developed for an X-Band gyro-TWA at the University of Strathclyde in 2007 (Cross et al., 2007). The methodology of the design was validated through results from numerical simulations, from MAGIC (MAGIC, 2002), agreeing well with the experimental results. A thermionic cusp gun was subsequently designed and numerically optimized based on this proven methodology. The MAGIC script used in this chapter is a derivative of the previous successful numerical code.

MAGIC allows different models of electron emission, for instance thermionic and explosive emission. The thermionic emission process was modeled using the Richardson-Dushman equation in Eq. 4.

$$
J_{e}=A_{e} T_{c}^{2} e^{\frac{-\phi_{w}}{k_{B} T_{c}}}
$$

where $T_{C}$ is the temperature of the emission surface and $k_{B}$ is the Boltzmann constant. The work function, $\phi_{w}$, was chosen to be $1.5 \mathrm{eV}$ - the value found for previous cathodes using a tungsten cathode impregnated with barium.

\subsection{Application requirements and design goals}

Two primary goals of the design of the cusp electron gun were: a) to produce an electron beam of suitable quality to drive the gyro-BWO over the required magnetic field range; and b) to produce a design simple enough that this could be manufactured with fewer complications compared with usual electron guns. Consideration of the construction of the diode played an important role in the design process, as the cathode would be small radially and thus sensitive to manufacturing tolerances. The aim was that a good quality electron beam would be produced even with some imperfections in cathode shape. The gyro-BWO parameters as-well-as electron beam power, voltage, current and $\alpha$ were found through beam-wave interaction simulation of the interaction region and analytical calculations of the dispersion profile (see section 3). The targeted performances of the electron gun and gyro-BWO are given in Table 1 . The axial velocity spread target of approximately less than $15 \%$ was chosen 
from previous investigation on the effect of velocity spread in helical waveguide gyro-devices (Denisov et al., 1998) where the velocity spread from $0 \%$ to $15 \%$ had little effect on the performance.

\begin{tabular}{|l|c|l|c|}
\hline \multicolumn{2}{|c|}{ Beam parameter targets } & \multicolumn{2}{c|}{ Gyro-BWO } \\
\hline Beam power & $60 \mathrm{~kW}$ & Max power $(\mathrm{CW})$ & $10 \mathrm{~kW}$ \\
\hline Accelerating voltage & $40 \mathrm{kV}$ & Efficiency & $17 \%$ \\
\hline Beam current & $1.5 \mathrm{~A}$ & Frequency band & $\mathrm{W}$-band \\
\hline Velocity ratio $(\alpha)$ & 1 to 2 & B-field range & $1.65-2.1 \mathrm{~T}$ \\
\hline Axial velocity spread & $<15 \%$ & Frequency tuning range & $84-104 \mathrm{GHz}$ \\
\hline
\end{tabular}

Table 1. Performance targets for the cusp electron gun and gyro-BWO.

The beginning of the design process focused on the emitting strip design and from this the focus electrodes and anode can be shaped around it. A schematic diagram of the general cathode geometry can be seen in Fig. 3, with some dimensions highlighted that are used in this discussion. The required dimensions of the emitter are: radial thickness of the strip, the average radius and the inclination of the surface. When a very narrow strip is chosen, a high quality beam can be produced, as the magnetic field variation - one of the leading causes of velocity and $\alpha$ spread - across the emission surface can be reduced at the expense of current density. Excessive current density, $>10 \mathrm{~A} / \mathrm{cm}^{2}$, can lead to a vastly reduced cathode lifetime; therefore, the thickness of the strip is chosen to produce a current density less than this limit. In this initial design stage this value was chosen to be approximately $8 \mathrm{~A} / \mathrm{cm}^{2}$.

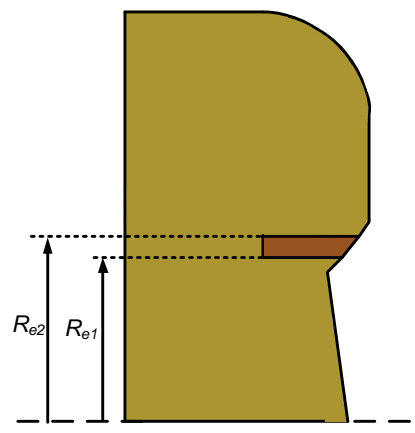

Fig. 3. Schematic diagram of the cusp electron gun cathode.

The emitting strip is inclined at an angle, as shown in Fig. 3. The average radius of the emitter can be chosen through the desired $\alpha$ value required. This is given through Eq. 5 .

$$
r_{c}=\frac{\alpha V_{0}}{\sqrt{\left(\alpha^{2}+1\right)\left|\omega_{c}\right| \omega_{0}}}
$$

The final design has the values of $R_{e 1}=5.79 \mathrm{~mm}, R_{e 2}=6.29 \mathrm{~mm}$ and emission current density $J_{c}=6 \mathrm{~A} / \mathrm{cm}^{2}$. A schematic diagram of the cusp gun geometry is shown in Fig. 4 with the Pierce principles (Pierce, 1954). 


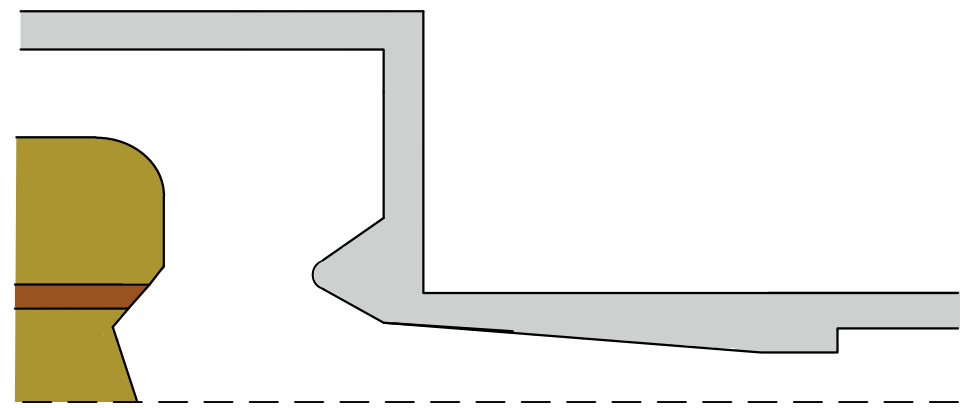

Fig. 4. Schematic of the simulation setup.

\subsection{Numerical simulations}

\subsubsection{Simulation parameters}

The geometry of the diode is simulated on a discrete spatial grid so this can lead to slight inaccuracies in the modeling when the mesh is not fine enough; however, if the system is meshed properly the results should be very accurate. The cathode can be visualized in both 2D, Fig. 5(a), and the full 3D, Fig. 5(b).

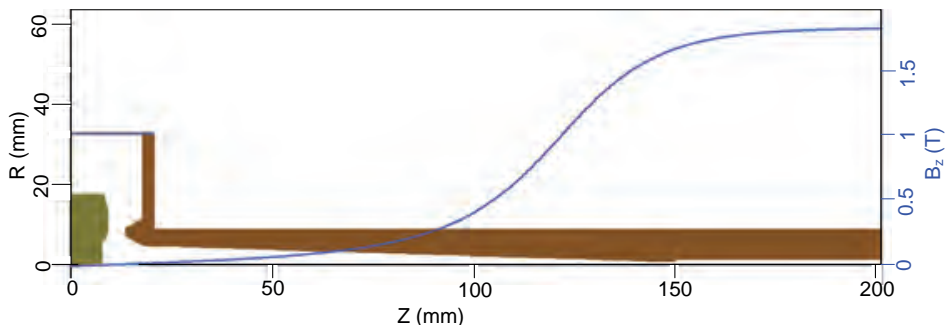

(a)

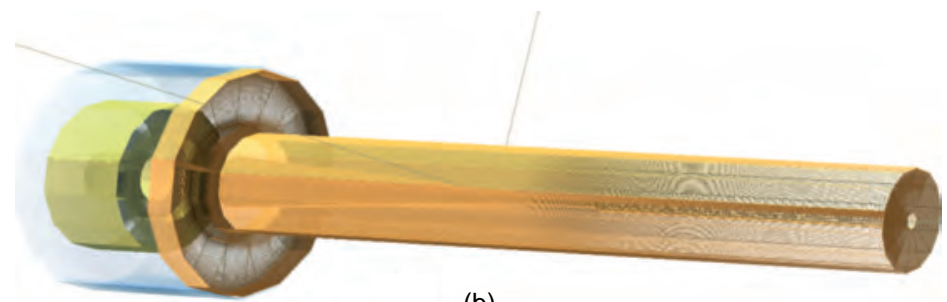

(b)

Fig. 5. Geometry of the cusp electron gun (a) 2D image with magnetic field profile overlaid and (b) 3D image.

It should be noted that although the simulation is $3 \mathrm{D}$, to decrease the run-time the electron beam was only emitted from 4 points around the 360 degree axis. This still yields accurate results, as the system is axially symmetrical. In Fig. 5(a), the magnetic field is overlaid showing the position of the cusp point in relation to the geometry of the cathode and anode. In Fig. 6 a more detailed view of the cathode, focusing electrodes and anode is shown. It should be noted that at the cathode there are two small gaps above and below the emitting surface (coloured brown). These gaps stop contact between the cathode and the focus 
electrodes, so that the barium in the cathode would not migrate into the focusing electrodes. Such migration could lead to unwanted electron emission from the focusing electrodes.

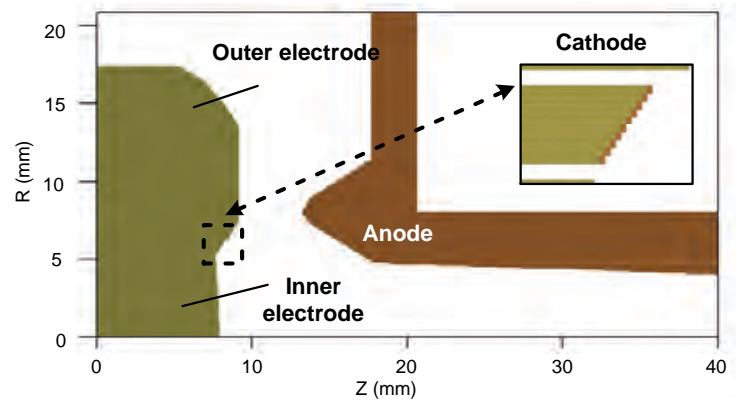

Fig. 6. A close-up view of the diode as illustrated by MAGIC.

\subsubsection{Simulation of B-field profile}

The magnetic coils defined in MAGIC are an approximation formed by a single line of coils at the average radius. The initial coil position is defined and then through a "do-loop" each subsequent coil is created. The magnetic field profile is critical to the operation of a cusp electron gun and the quality of the electron beam. An extra coil, so-called "shim coil", was added to each end of the cavity solenoid. The shim coils sharpen the magnetic field profile and reduce the total length of the solenoid and lower the electrical power consumption. The parameters of the solenoids are given in Table 2.

\begin{tabular}{|l|c|c|c|c|}
\hline & Reverse coil & Cavity coil & $\mathbf{1}^{\text {st }}$ Shim coil & $2^{\text {nd }}$ Shim coil \\
\hline Start position & $-6 \mathrm{~cm}$ & $12.3 \mathrm{~cm}$ & $12.3 \mathrm{~cm}$ & $31.6 \mathrm{~cm}$ \\
\hline Average radius & $8 \mathrm{~cm}$ & $2.84 \mathrm{~cm}$ & $4.92 \mathrm{~cm}$ & $4.92 \mathrm{~cm}$ \\
\hline Wire width & $2.2 \mathrm{~mm}$ & $2.2 \mathrm{~mm}$ & $2.2 \mathrm{~mm}$ & $2.2 \mathrm{~mm}$ \\
\hline Number of turns & 10 & 103 & 15 & 15 \\
\hline Coil current & $713.28 \mathrm{~A}$ & $3257.8 \mathrm{~A}$ & $465.4 \mathrm{~A}$ & $465.4 \mathrm{~A}$ \\
\hline
\end{tabular}

Table 2. Properties of the solenoids defined in MAGIC simulation code

It is important to note that while the current of the reverse coil is equal to $713.28 \mathrm{~A}$ in each turn, in practical terms, this would be distributed over a 4 layer coil with $178.32 \mathrm{~A}$ per layer. Similarly, for the cavity coil $3257.8 \mathrm{~A}$ is equal to 14 layers of 232.7 A per layer. The region of the flat top magnetic field strength has to match the length of the helically corrugated waveguide. The full magnetic field profile of the solenoid is shown in Fig. 7.

The magnetic fields at the cathode and the cavity solenoids are adjustable factors that determine the $\alpha$ value of the electron beam in the beam-wave interaction region. The spread of magnetic field over the emission surface is one of the biggest factors that contributes to velocity spread in the electron beam. The magnetic field vectors in the cusp region are shown in Fig. 8. This shows the direction and amplitude of the magnetic field that the electrons travel through from the cathode to the anode aperture. It also shows the position of the cusp point, in this case, at $4.3 \mathrm{~mm}$ from the middle point of the front face of the emitter. 


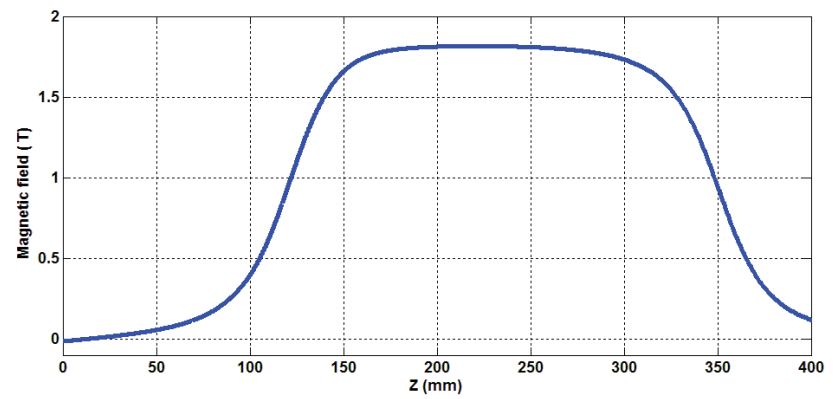

Fig. 7. Axial magnetic field $\left(B_{\max }=1.82 \mathrm{~T}\right)$ along axis of symmetry as calculated by MAGIC.

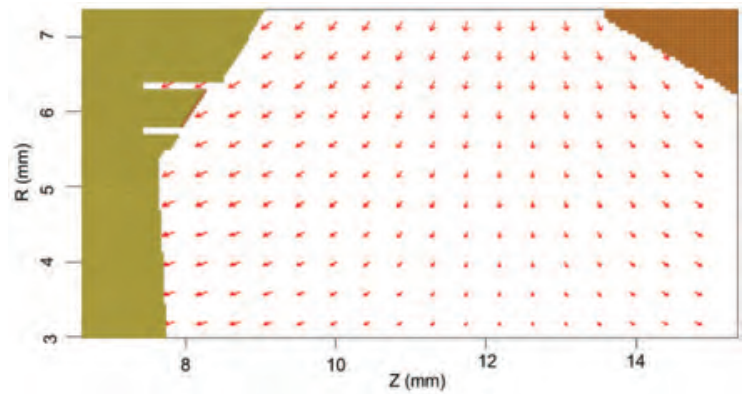

Fig. 8. Magnetic field vectors in the cathode-anode region.

\subsubsection{Equipotential surfaces and electric field enhancement}

The electron beam is focused as a result of the shape of the equipotentials in the diode region. The equipotentials are controlled through the shape of the focusing electrodes and the anode. The equipotentials in the diode region, Fig. 9(a), show us how the electrons are focused by the shape of the anode and cathode surface. The inner and outer focusing electrodes are used to convey the electron beam into the anode aperture.

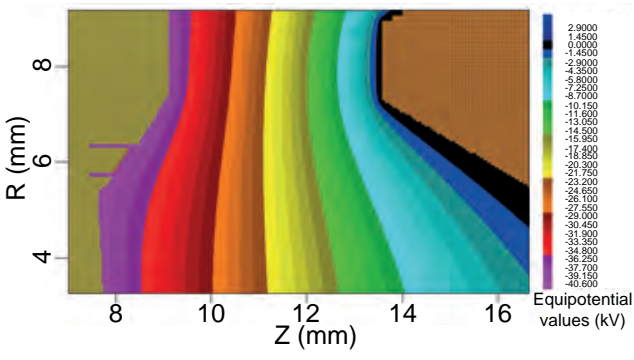

(a) Equipotentials

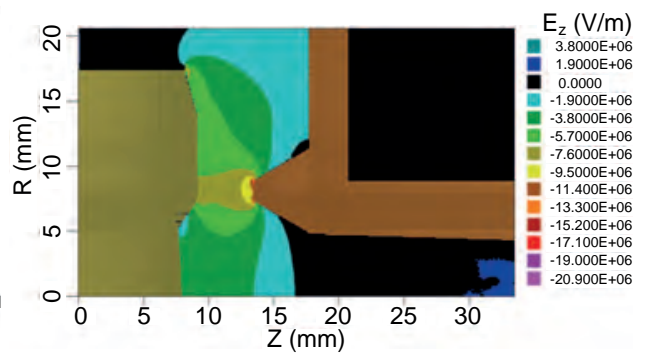

(b) $E_{z}$

Fig. 9. Electric field profile in the cathode-anode region.

The magnitude of the electric field at the cathode and anode gap is a concern for electron gun design due to the possibility of electric field breakdown. The electric field at the cathode surface is required to be lower than the breakdown threshold in vacuum in order to ensure the 
cathode is not damaged during operation. The axial electric field $\left(E_{z}\right)$ when the accelerating voltage is at its maximum $40 \mathrm{kV}$ was recorded and is shown in Fig. 9(b). This field was below the breakdown threshold of $10 \mathrm{MV} / \mathrm{cm}$. When the cathode is constructed the sharp edges would be rounded and so the areas of high electric field would be reduced.

\subsection{Simulated electron trajectories}

The electron trajectories after emission from the cathode are one of the most important diagnostic tools as these show if the electrons pass through the beam tube, where possible interception may occur, the thickness of the electron beam at the plateau magnetic field region and if the electron beam can pass through the backstop filter (the smallest diameter area of the tube). The electron trajectories through the diode and into the downstream uniform B-field region are shown in Fig. 10(a). These pictures show that the electrons pass through the waveguide geometry and form an axis-encircling beam, a view of which is clear to see in Fig. 10(b). There is small quantity of reflected electrons shown in this trajectory plot. These electrons amount to less than $1 \mathrm{~mA}$, compared to the electron current of $1.5 \mathrm{~A}$. At the end of the beam tube the thickness of the electrons beam can be calculated from the electron trajectories at the point of maximum magnetic field. The exact properties of this beam can be seen in Table 3. This shows that this beam has a thickness of $\sim 0.2 \mathrm{~mm}$ corresponding to a spread of $60 \%$.
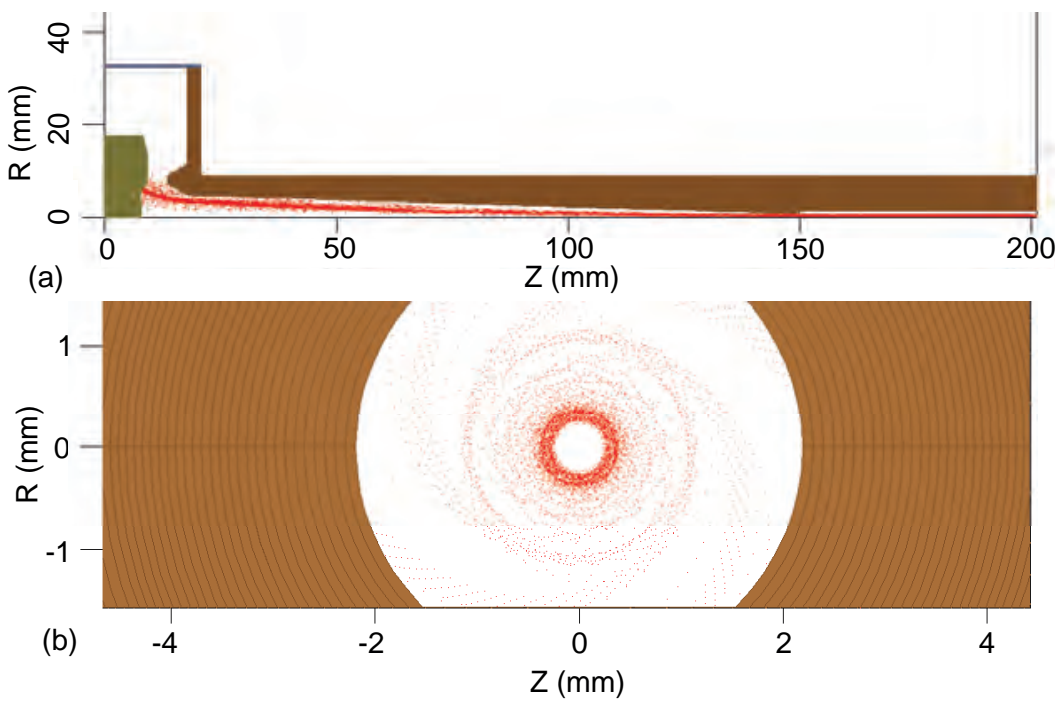

Fig. 10. MAGIC simulated electron trajectories at $1.82 \mathrm{~T}$ cavity field showing (a) the beam in the $r-z$ plane with the full geometry and (b) cross-sectional shape at the downstream region.

\subsubsection{Electron beam current and voltage}

The design of the waveguide interaction region determined the required electron beam current and energy. Simulation of these properties along the waveguide allows one to measure the beam power produced by the electron gun and compare that to the ideal target set for the electron gun. In the simulation a slowly rising accelerating voltage pulse was applied to the cathode and focusing electrodes. The rise time of the pulse was $1 \mathrm{~ns}$ with a steady voltage 


\begin{tabular}{|l|c|}
\hline Minimum radius & $0.229 \mathrm{~mm}$ \\
\hline Maximum radius & $0.421 \mathrm{~mm}$ \\
\hline Average radius & $0.325 \mathrm{~mm}$ \\
\hline Radius spread $(\Delta r / r)$ & $59.2 \%$ \\
\hline Envelope ripple & $15 \%$ \\
\hline
\end{tabular}

Table 3. Properties of the electron trajectories at the magnetic field plateau region, $B_{z}=1.82 \mathrm{~T}$.

after that time. The current emitted from the cathode was simulated to be $1.57 \mathrm{~A}$. This value was found to be $1.5 \mathrm{~A}$ emitted from the face of the emitter and $0.07 \mathrm{~A}$ from inside the gap between the emitting surface and the focusing electrode, which was not transported along the beam tube. The electron beam current at the downstream uniform magnetic field is a vital diagnostic as this allows calculation of the transported electron beam current to show what percentage of the electron beam is reflected or transmitted. The measured current downstream corresponded to $99.9 \%$ of the beam emitted.

\subsubsection{Pitch angle and axial velocity spread}

The two parameters of the electron beam that determine the interaction strength and efficiency of the gyro-BWO are the spreads in $\alpha$, and axial velocity. The $\alpha$ value of the electron beam is a measure of the ratio of perpendicular to parallel velocity $\alpha=v_{\perp} / v_{\|}$(He et al., 2001). Since it is only the transverse velocity, that participates in the interaction, this is a measure of the amount of the electron beam energy that is available for the interaction. The axial velocity spread will result in broadening of the electron cyclotron frequency and therefore excessive axial velocity spread will give rise to low beam-wave interaction efficiency.

The $\alpha$ value sought is variable between 1 and 2 but centered on $\sim 1.65$. The $\alpha$ value as a function of the axial length along the beam tube was observed in the simulation and is shown in Fig. 11. Clearly shown here is the rise in the $\alpha$ value along the waveguide tube due to the rise in magnetic field. There are two stray beam lines shown here with a very large $\alpha$ value. These are emitted from inside the cathode gap and for the purpose of these calculations are not taken into consideration when estimating the average $\alpha$ value, and its spread. The calculated $\alpha$ values can be seen in Table 4 showing a spread of $10.7 \%$.

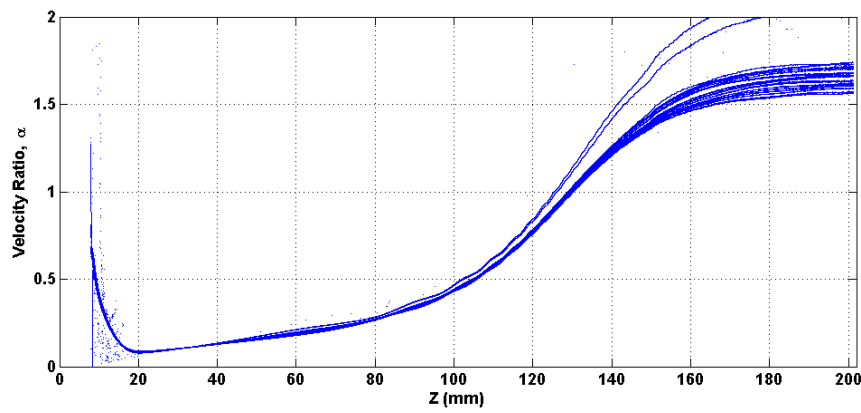

Fig. 11. Simulated $\alpha$ of the electron beam as a function of axial position. Measured with a magnetic field at the uniform downstream region of $B_{z}=1.82 \mathrm{~T}$.

The axial momentum of the electrons (normalized to the electron rest mass $m_{e}$ ) along the axial ordinate is shown in Fig. 12. This shows the trajectories of the two electron beamlets with a 


\begin{tabular}{|l|c|}
\hline Minimum $\alpha$ value & 1.56 \\
\hline Maximum $\alpha$ value & 1.74 \\
\hline Average $\alpha$ value & 1.65 \\
\hline$\alpha$ spread $(\Delta \alpha / \alpha)$ & $10.7 \%$ \\
\hline
\end{tabular}

Table 4. Simulated $\alpha$ values at $B_{z}=1.82 \mathrm{~T}$.

much lower axial momentum than the rest of the electron beam consistent with the simulated results for $\alpha$. If it is assumed that there is a negligible difference in electron mass from the lower and upper values of the momentum then the axial velocity spread can be found from Eq. 6.

$$
\frac{\Delta \mathrm{v}_{z}}{\mathrm{v}_{z, a v}}=\frac{\Delta m \mathrm{v}_{z}}{m \mathrm{v}_{z, a v}}=\frac{\Delta P_{z}}{P_{z, a v}}
$$

where $P_{z}$ is axial momentum, $P_{z, a v}$ is average axial momentum, $\mathrm{v}_{z}$ is axial velocity and $\mathrm{v}_{z, a v}$ is average axial velocity.

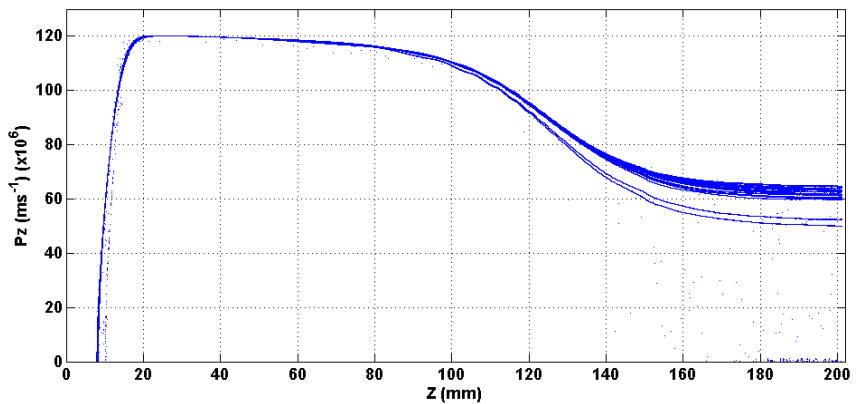

Fig. 12. Simulated axial momentum of the electron beam as a function of axial position.

Analysis of the axial momentum at the maximum magnetic field allows calculation of the axial velocity spread and the values obtained are given in Table 5 . The axial velocity spread is within the design target.

\begin{tabular}{|l|c|}
\hline Minimum axial velocity value & $5.97 \times 10^{7} \mathrm{~ms}^{-1}$ \\
\hline Maximum axial velocity value & $6.47 \times 10^{7} \mathrm{~ms}^{-1}$ \\
\hline Average axial velocity & $6.22 \times 10^{7} \mathrm{~ms}^{-1}$ \\
\hline Axial velocity spread $\left(\Delta v_{z} / v_{z}\right)$ & $8.1 \%$ \\
\hline
\end{tabular}

Table 5. Values of axial momentum and corresponding axial velocity spread at the plateau magnetic field region, $B_{z}=1.82 \mathrm{~T}$.

\subsubsection{Variation of magnetic field and different combinations of electron beam properties}

The interaction frequency can be tuned through adjusting parameters of the electron beam such as accelerating voltage, $\alpha$ as well as the cavity magnetic field strength. In order to change the $\alpha$ values of the electron beam, the magnetic field at the cathode can be varied. The $\alpha$ value as a function of magnetic field at the cathode is shown in Fig. 13 at a fixed cavity magnetic field of 1.82 T. The $\alpha$ value can be analytically calculated through Eq. 3 and agrees well with 
the simulated value. The effect of varying the $\alpha$ value changes the electron beam dispersion line so different interaction frequencies can be achieved.

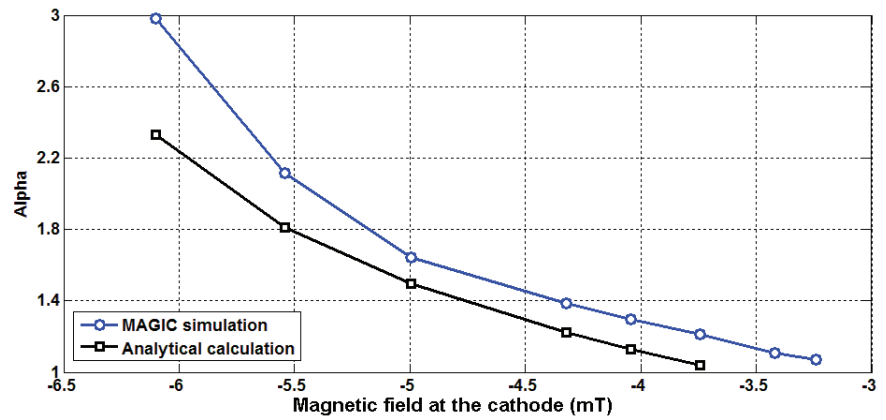

Fig. 13. $\alpha$ value as a function of magnetic field at the cathode surface. The cavity magnetic field is kept constant at $B_{z}=1.82 \mathrm{~T}$.

While the cathode magnetic field is adjusted in order to change the value of $\alpha$ over the range of 1 to 3 , the electron beam qualities ( $\alpha$ and axial velocity spreads) will be affected. This can be seen in Fig. 14 where the optimum electron beam qualities are obtained at the designed cathode magnetic flux density of $-4.97 \mathrm{mT}$ corresponding to an $\alpha$ of 1.65 .

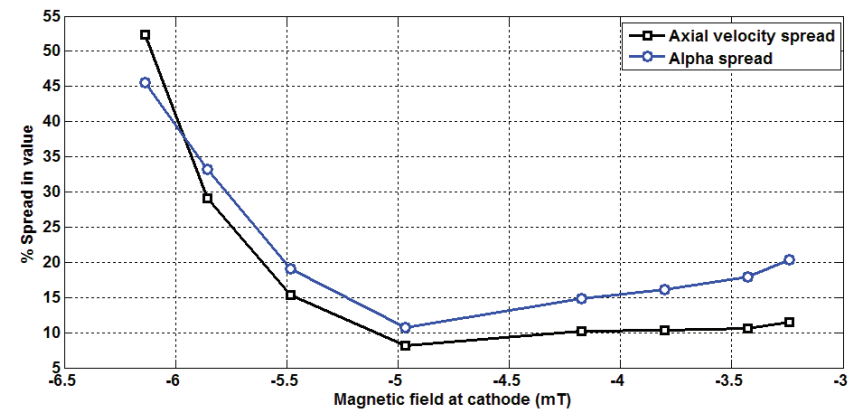

Fig. 14. Simulated values of axial velocity and $\alpha$ spreads as a function of cathode magnetic field strength.

The designed beam voltage is $40 \mathrm{kV}$ but the gyro-BWO is tunable in frequency when the voltage changes so the electron gun had to transport the beam through the drift tube with an acceptable beam quality over a range of voltages. It was found that when the beam voltage varied from $35-45 \mathrm{kV}$ the large orbit beam was still fully transported to the downstream cavity region. However, with a constant magnetic field configuration the beam $\alpha$ would be different and so some adjustment in the reverse coil strength would have to be made through the range of voltages. The $\alpha$ value was 2.46 and 1.35 for $35 \mathrm{kV}$ and $45 \mathrm{kV}$ respectively.

The electron beam quality also varies over this voltage range, as shown in Fig. 15. The plateau of the electron beam quality curve is centered on the designed voltage of $40 \mathrm{kV}$ showing the optimized electron gun design. Throughout the range of voltages the electron beam maintained an acceptable quality, defined by the axial velocity and $\alpha$ spreads. 


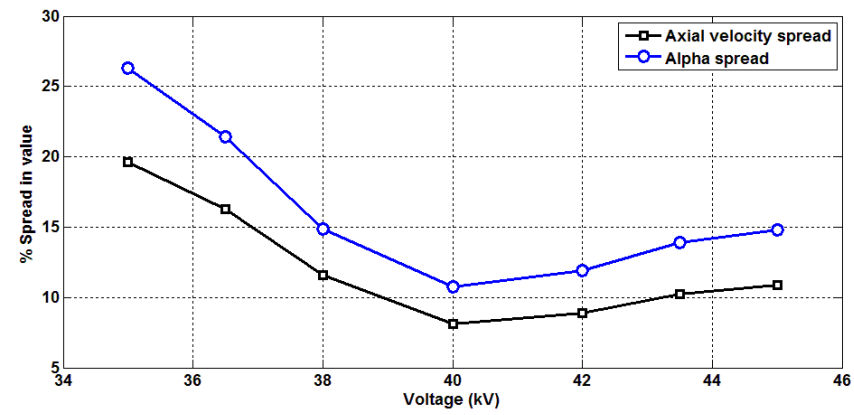

Fig. 15. Simulated axial velocity and $\alpha$ spreads as a function of the beam voltage. The average $\alpha$ and cavity magnetic field is kept constant at 1.65 and $1.82 \mathrm{~T}$ respectively.

If a constant $\alpha$ value is required when the voltage is changed the cathode magnetic field, and so reverse coil current, would have to be adjusted for each value of accelerating voltage. The range of cathode magnetic field that kept $\alpha$ at 1.65 with a cavity magnetic field of $1.82 \mathrm{~T}$ is shown in in Fig. 16.

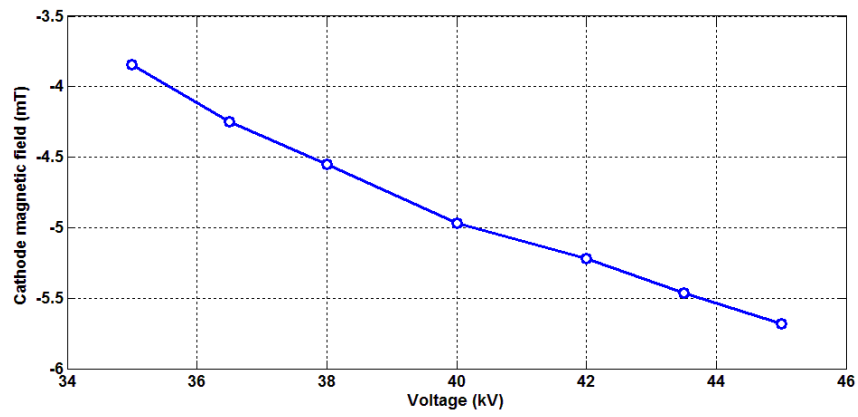

Fig. 16. Variation in cathode magnetic field required to keep a constant $\alpha(=1.65)$ as the applied voltage is swept from $35 \mathrm{kV}$ to $45 \mathrm{kV}$.

Changing the cavity magnetic field strength allows the frequency of interaction to be changed to any desired frequency over the full range of the gyro-BWO interaction, $84-104 \mathrm{GHz}$. This has a stronger effect on the electron beam line than any other method of frequency adjustment. The axial velocity and $\alpha$ spreads were simulated in the operating cavity magnetic field region and is shown in Fig. 17. Since the geometry of the cusp electron gun was optimised for the centre frequency of $94 \mathrm{GHz}$ i.e. at a magnetic field of $1.82 \mathrm{~T}$, the simulation at a different magnetic field would be an un-optimised setup so by changing some of the variables such as the reverse coil position, cavity coil position and applied voltage these results can be improved.

To obtain a constant value of $\alpha$ in the gyro-BWO operating regime which required a cavity magnetic field of $1.65 \mathrm{~T}-2.1 \mathrm{~T}$ the cathode magnetic field must be changed in accordance with the change in the cavity magnetic field. The value of the magnetic field at the cathode as a function of the cavity magnetic field required to generate an $\alpha$ value of 1.65 is shown in Fig. 18. 


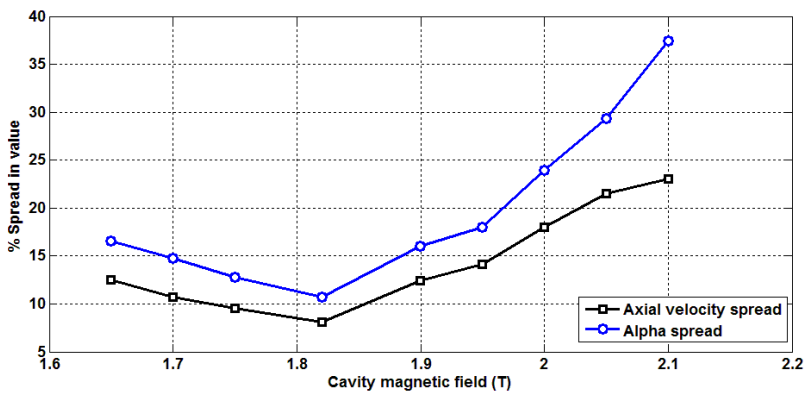

Fig. 17. $\alpha$ and axial velocity spreads as the cavity magnetic field is swept from $1.65 \mathrm{~T}-2.1 \mathrm{~T}$. The magnetic field at the cathode is adjusted in order to keep the $\alpha$ constant at 1.65.

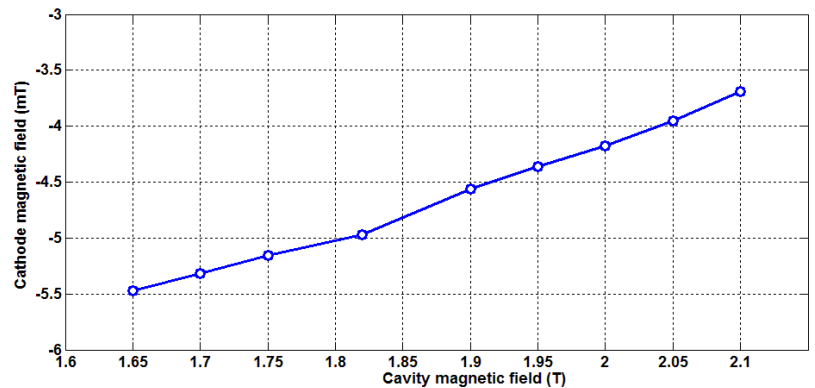

Fig. 18. Values of the magnetic field at the cathode required to keep a constant $\alpha(=1.65)$ as the cavity magnetic field strength is changed.

\subsection{Summary}

In this section the cusp electron gun was designed, simulated and optimized to produce an axis-encircling annular beam of $40 \mathrm{kV}, 1.5 \mathrm{~A}$ with an $\alpha$ of 1.65. The design was originally simulated in MAGIC and optimized through changing the diode geometry (Donaldson et al., 2010). The optimized design produced an electron beam with low axial velocity and $\alpha$ spreads at the center magnetic field strength with acceptable quality over the full magnetic field tuning range. Other factors were investigated for instance the scope for tuning $\alpha$ in the range of 1 to 2 and the potential for voltage tuning of the output frequency. In each case the electron beam passed through the beam tube without scraping or mirroring and had tolerable spreads in axial velocity and $\alpha$ spreads. The optimized electron gun design produced an electron beam of high enough quality in order to drive the beam-wave interaction within the gyro-BWO (Donaldson et al., 2009; Li et al., 2010).

\section{Beam-wave interaction in gyro-BWO}

\subsection{Background}

The surface of the helically grooved waveguide of the gyro-BWO can be represented in cylindrical coordinates $r, \phi, z$ as follows

$$
r(\phi, z)=r_{0}+l \cos (\bar{m} \phi+\bar{k} z)
$$


where $r_{0}$ is the waveguide mean radius, $l, \bar{m}$ and $\bar{k}=2 \pi / d$ are the amplitude, azimuthal and axial numbers of the corrugation respectively, and $d$ is the corrugation period. If a three-fold helical waveguide is used $(\bar{m}=3)$ the corrugation would provide effective coupling of the $\mathrm{TE}_{21}$ near cut-off mode and the $\mathrm{TE}_{11}$ traveling mode if the corrugation period is chosen so that the Bragg conditions

$$
\bar{k} \approx k_{11}, m_{A}+m_{B}=\bar{m}
$$

are satisfied, where $k_{11}$ is the axial wavenumber of the $\mathrm{TE}_{11}$ mode at the cutoff frequency of the $\mathrm{TE}_{21}$ mode and $m_{A}$ and $m_{B}$ are the azimuthual index of the near cutoff and traveling modes respectively.

The resonant coupling of the waves corresponds to the intersection of their dispersion curves or, more exactly the intersection between the $\mathrm{TE}_{21}$ mode and the first spatial harmonic of the $\mathrm{TE}_{11}$ mode (Fig. 19) and would result in an eigenwave with a $\mathrm{TE}_{21}$-like cross-sectional electric field distribution. For such a field structure it is favourable to use the second harmonic of the electron cyclotron frequency for beam-wave interaction, which has the advantage of lowering the required magnetic field strength by a factor of two. The axis-encircling beam resonantly excites only co-rotating $\mathrm{TE}_{n m}$ modes with azimuthal indices equal to the cyclotron harmonic number, $\bar{m}=s$. The helical symmetry allows transformation of a selected direction of azimuthal rotation to a selected axial direction, in this case a wave which is propagating in a counter direction with respect to the electrons' axial velocity. The electron beam's linear dispersion characteristic can be adjusted with respect to the wave dispersion over a rather broad frequency range by changing either the axial guide magnetic field or the electron accelerating potential.

\subsection{Dispersion and linear theory}

The resonant coupling of the waves corresponds to the intersection of their dispersion curves. If the amplitude of the corrugation is small compared with the wavelength, the dispersions of the resultant eigenwaves, i.e. $w_{1}$ and $w_{2}$ in the helical waveguide, can be calculated approximately by the following equation from analytical perturbation theory (Denisov et al., 1998)

$$
\left(h^{2}-2 \delta\right)\left(h-\Delta_{g}+\delta / h_{0}\right)+2 \sigma^{2} / h_{0}=0
$$

where all the symbols (also those that appear later) retain the meanings defined in ref. (Denisov et al., 1998). One of the eigenwaves, i.e. $w_{1}$, having a near constant negative group velocity and small axial wavenumbers in the designed operating frequency range, is the operating eigenwave of the interaction.

The electron cyclotron mode, normalized in a manner consistent with Denisov et al 1998, can be written as

$$
\delta-h \beta_{z 0}=s \Delta_{H}
$$

The output frequency of the gyro-BWO interaction can therefore be calculated from the intersection of the dispersions of the eigenwaves and the beam cyclotron mode (Fig. 20).

For the highest interaction efficiency the gyro-BWO should be operated in a region of small axial wavenumber so that the detrimental effect of the Doppler broadening of the electron cyclotron line because of spread in axial electron velocity is minimized. Therefore a larger gradient of the eigenwave $w_{1}$ is favourable for increasing the interaction efficiency and frequency tuning range. For a gyro-BWO using a smooth cylindrical waveguide, the backward wave exists only in the negative half of the axial wavenumber, but for the 


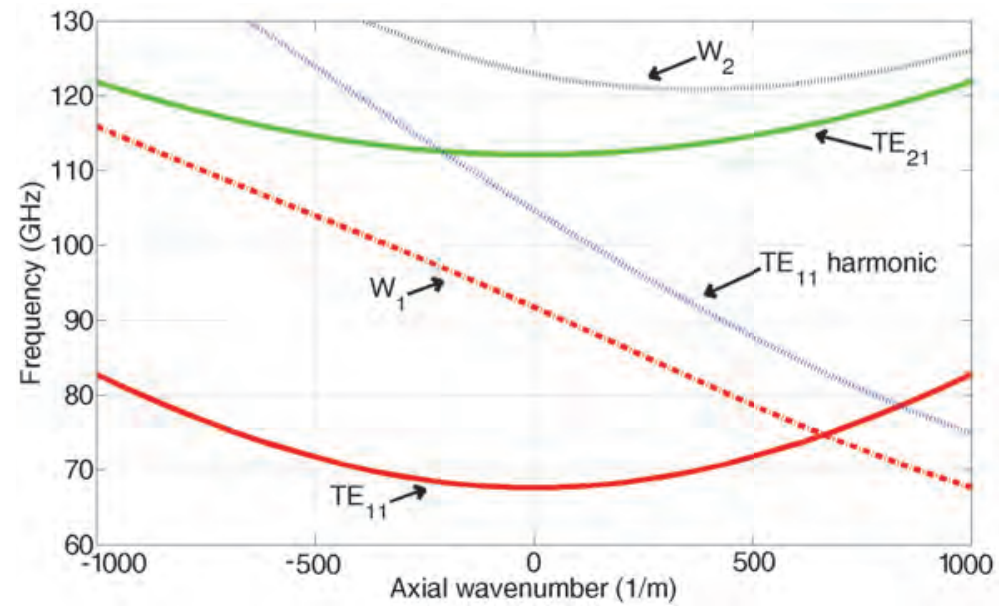

Fig. 19. Mode coupling between the spatial harmonic $\mathrm{TE}_{11}$ mode and $\mathrm{TE}_{21}$ mode.

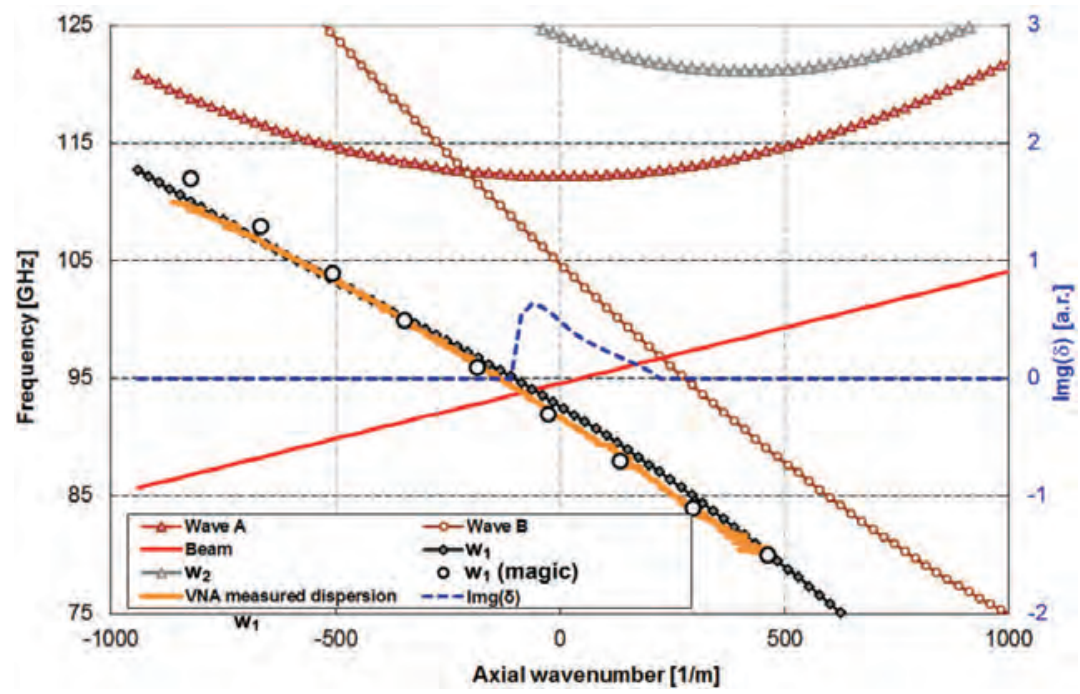

Fig. 20. The operating dispersion of the helical waveguide.

eigenwave $w_{1}$ in the helical waveguide the backward wave exists in both the negative and positive range of wavenumbers. Therefore the frequency tuning range of a gyro-BWO using a helical waveguide would have much wider frequency tuning range compared with its smooth-bore counterpart. The gradient of $w_{1}$ can be adjusted by altering the period and the corrugation amplitude of the helical waveguide.

In analogy to gyrotron interactions and ref. (Denisov et al., 1998), it is possible for one to derive the gyro-BWO beam-wave dispersion 


$$
\begin{aligned}
& {\left[\left(h^{2}-2 \delta\right)\left(h-\Delta_{g}+\delta / h_{0}\right)+2 \sigma^{2} / h_{0}\right]\left[h-\left(\delta-\Delta_{H}\right) / \beta_{z 0}\right]^{2}} \\
& =C^{3}\left(h-\Delta_{g}+\delta / h_{0}\right)\left\{1+\frac{2 s}{\alpha_{0}^{2} \beta_{z 0}}\left[h-\left(\delta-\Delta_{H}\right) / \beta_{z 0}\right]\right\}
\end{aligned}
$$

where $\alpha_{0}$ and $\beta_{z 0}$ are the beam initial pitch angle and relative velocity in the longitudinal direction respectively. The interaction frequency of the gyro-BWO can be calculated by solving the uncoupled beam-wave equation by setting $C=0$ in Eq. 11, i.e. the intersection of the eigenwave $w_{1}$ and the beam dispersion line. In a general case, Eq. 11 has four $\delta(h)$ roots, with two real roots being the "hot" (electron beam present) eigenwaves, and a pair of conjugate complex roots, which are degenerates of the electron cyclotron mode due to the CRM interaction at and near the intersection when the beam parameters are suitably chosen. The negative imaginary number of the solution (Fig. 20, dashed line showing one such interaction for the gyro-BWO) gives rise to the oscillation that grows with time in the cavity and hence allows the starting condition and the small signal growth of the oscillation to be analyzed.

The dispersion of the operating eigenwave can be found by measuring the phase evolution of a counter-rotating circularly polarized wave when it propagates through the waveguide by using a vector network analyzer (VNA). It can also be measured by detecting the polarization angle of a linearly polarized wave when it propagates through the waveguide by using a scalar network analyzer (SNA)(Burt et al., 2004). In Fig. 20 the measured results using the VNA method are shown and compared with the results simulated by MAGIC using the same operating eigenwave. In the simulation using the MAGIC code, a left-polarized circular wave of one frequency was injected into the right-hand helical waveguide, and a component of the electric field inside the waveguide was measured along the axial direction. The measured field was then numerically analyzed and the axial wavenumber of the eigenwave was therefore obtained for that frequency (He et al., 2011).

\subsection{Simulation of the beam-wave interaction}

The dimensions of the helical structure used in this simulation were designed to support an operating eigenwave of a higher group velocity to achieve a higher electronic efficiency and wider frequency tuning range. MAGIC simulation of the performance of the gyro-BWO using this helical waveguide as the interaction region when driven by an electron beam of energy $40 \mathrm{keV}$, current $1.5 \mathrm{~A}$ and $\alpha 1.65$ are presented.

The radiation of the gyro-BWO can be coupled out at two positions; One from an output coupler at the upstream side of the corrugated waveguide, the other through an output window at the downstream end. In the latter case the output window will act as a boundary of the cavity and therefore some reflection from the window (which can be as low as 1\%) is desirable for the oscillation to start. Both the simulations and previous experiments at X-band (He et al., 2005) confirmed that the performance of the gyro-BWO is the same when using the two different output methods. In the simulation of the $\mathrm{W}$-band gyro-BWO, the output power resulted from the beam-wave interaction in the helical waveguide region which was absorbed by a "microwave absorber" at the upstream location. This was achieved by defining a region of finite conductance as shown in Fig. 21. The output power of the gyro-BWO could therefore be simulated by measuring the total ohmic loss in this conductive volume. It was found in the simulation that the electron beam parameters are unaffected by this region.

An electron beam with parameters similar to that simulated and measured in the experiment was used, i.e. beam energy, current and beam pitch angle, guided by a magnetic field of 


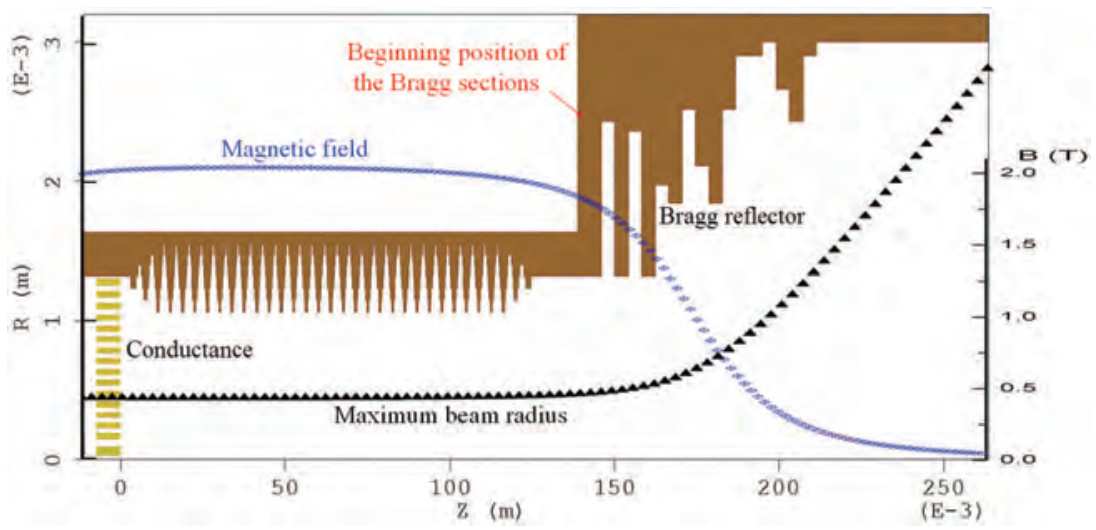

Fig. 21. The geometry used to simulate the beam-wave interaction.

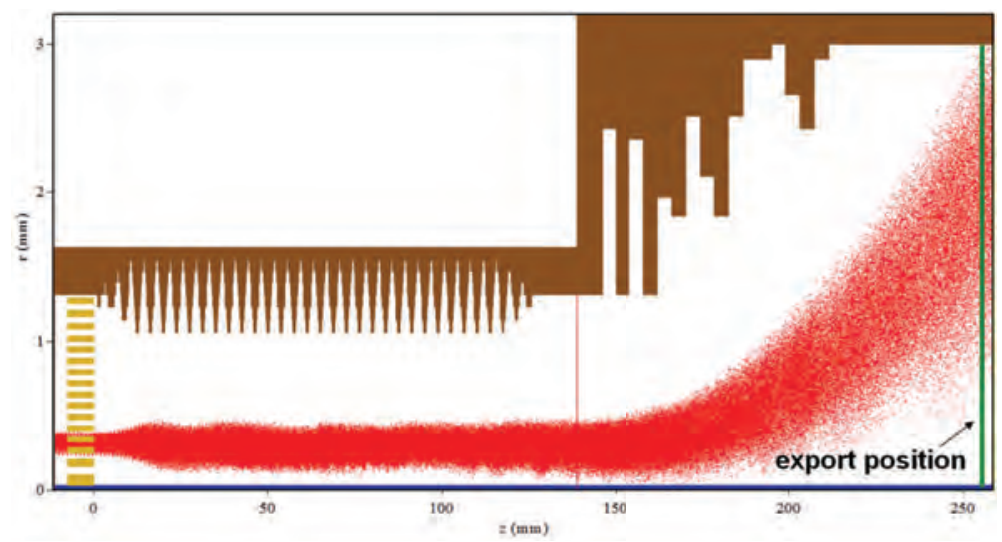

Fig. 22. The electron beam trajectories of an axis-encircling solid beam.

about $2.1 \mathrm{~T}$ in a beam tube of radius $1.3 \mathrm{~mm}$ in which the lowest order mode was cut-off and propagating in the downstream (right) direction. For the gyro-BWO to oscillate, the electron beam should rotate in the opposite direction to the helical structure. At the downstream end, a Bragg reflector was used to completely reflect any microwave signal back into the interaction region. At the same time the spent electron beam can pass through the Bragg reflector region. The electron beam (with all its physical parameters and the electromagnetic wave (with all its parameters) were then recorded at the cross-sectional plane located downstream from the Bragg reflector. This allowed the simulation of the depressed collector for energy recovery purpose as discussed in the later sections. A snap shot of the simulation showing the geometry and electron beam trajectories of the axis-encircling solid beam that was used in the experiment is shown in Fig. 22. When the magnetic field was $1.82 \mathrm{~T}$, electron beam energy $40 \mathrm{keV}$, current $1.5 \mathrm{~A}$, pitch $\alpha 1.65$, a simulated power of $10 \mathrm{~kW}$ and frequency of $94 \mathrm{GHz}$ were obtained. A typical simulated output spectrum and mode pattern are shown in Fig. 23. The simulated output power as a function of frequency is shown in Fig. 24. A 3 dB tuning range of $84-104 \mathrm{GHz}$ was predicted from the simulation of the W-band gyro-BWO. 
FIELD Magnitude Of FFT of Ephi at RECEND

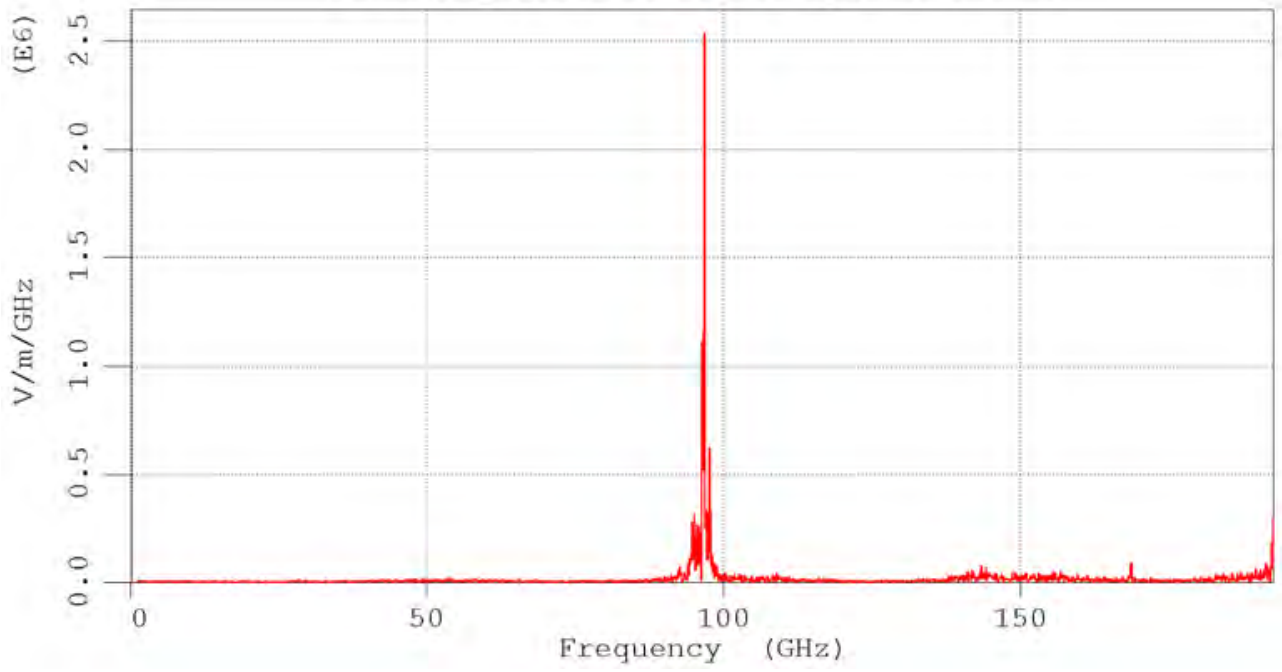

(a) Output spectrum of the gyro-BWO.

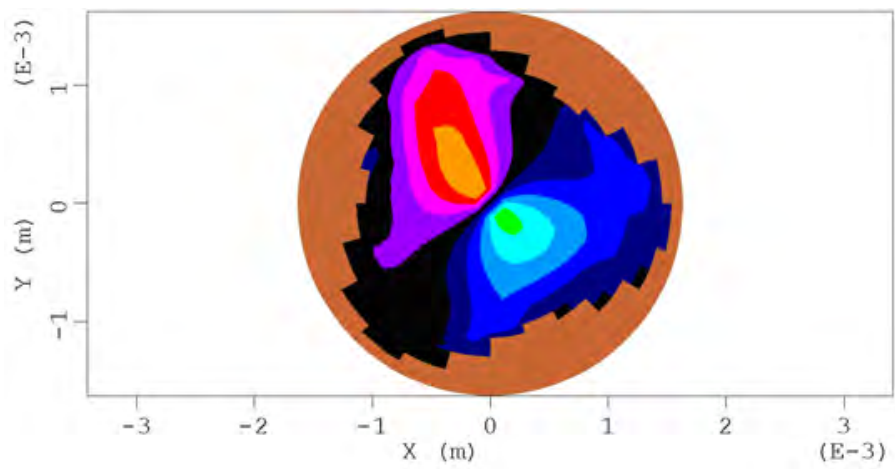

(b) E-field distribution inside the HCIR.

Fig. 23. Characteristic of microwave output from the gyro-BWO.

\section{Simulation of the depressed collector}

\subsection{Principle of the depressed collector}

The overall efficiency is an important parameter for high-power microwave sources. For a given RF output power, higher efficiency means less primary power is needed. Microwave sources with higher efficiency have less heat dissipation which means smaller cooling systems are needed. High efficiency is essential in space applications and some ground-based applications, such as deep space communication and mobile installations.

Several methods have been developed to improve the efficiency of the beam-wave interaction. One is to change the profile of the waveguide to obtain a higher electronic efficiency, such as employing a slot structure, helical structure as used in this chapter, slice structure, and so 


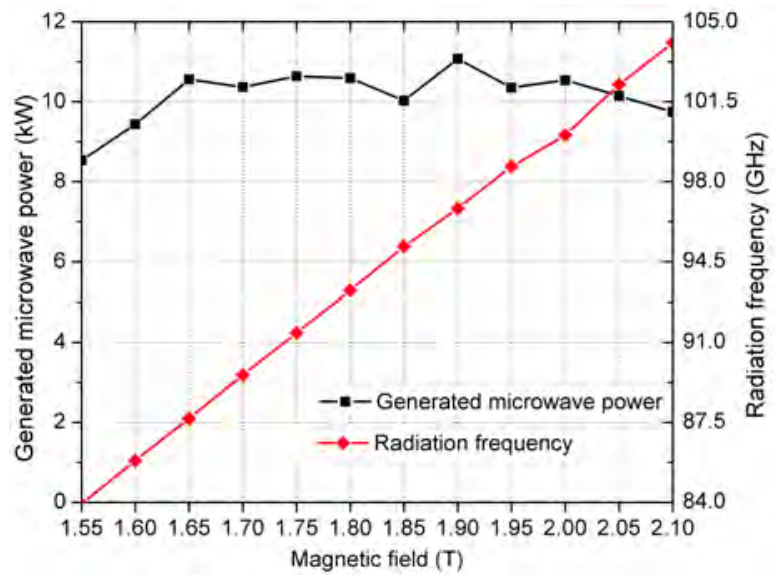

Fig. 24. The output powers and frequencies at different cavity magnetic fields.

on. The other way is to use a tapered magnetic field or tapered wall radius instead of the constant ones. (Ganguly \& Ahn, 1989; Nusinovich \& Dumbrajs, 1996; Sprangle \& Smith, 1980; Walter et al., 1996) Another option for enhancing the efficiency is to recover energy from the spent beam using single or multi-stage depressed collectors. It has been shown that this is an effective way to improve the overall efficiency of microwave tubes, such as conventional klystrons, BWOs and TWTs (Neugebauer \& Mihran, 1972; Wilson et al., 2007).

Depressed collectors are passive converters that can transfer the kinetic energy of the spent electrons into potential electric energy. "Depressed"means that the collector has a depressed potential as compared with the main body of the tube. The electrons lose their kinetic energy when passing through the retarding electrostatic field and finally land on the collector surface with a significant reduction in kinetic energy. They produce a loop current which results in a power recovery from the spent electrons (Sterzer \& Princeton, 1958). The collected power by a depressed collector can be written as

$$
P_{c o l}=\sum_{n=1}^{N} V_{n} I_{n}
$$

Here $N$ is the number of stages and $V_{n}, I_{n}$ are the potentials and collected current on the $n^{\text {th }}$-stage electrode, respectively. For a given energy distribution of the spent electrons, increasing the number of stages results in the collection of more power. However, the design of depressed collectors becomes more complex and the cost increases as the number of stages increases.

By introducing a depressed collector with a collection efficiency of $\eta_{c o l}=P_{\text {col }} / P_{\text {spent_beam }}$ and output efficiency $\varepsilon_{\text {out }}$ which is the ratio of $P_{\text {out }}$ and the total microwave power in the cavity, the overall efficiency of the microwave tube with an electronic efficiency $\eta_{e}$ can be calculated using

$$
\eta_{\text {tot }}=\frac{P_{\text {out }}}{P_{b}-P_{\text {col }}}=\frac{\varepsilon_{\text {out }} \eta_{e}}{1-\eta_{\text {col }}\left(1-\eta_{e}\right)}
$$


For those inherently low efficiency high power microwave devices, depressed collectors with efficiencies higher than $80 \%$ can significantly improve the overall efficiencies. For a moderately efficient source with an electronic and collection efficiency of $30 \%$ and $80 \%$, respectively, with the use of depressed collection the overall efficiency could be increased to $61.4 \%$ when $\varepsilon_{\text {out }}=0.9$, increasing the overall efficiency by a factor of 2 .

To design a depressed collector with high efficiency, several issues need to be considered before commencing simulations.

a) Determining the potentials and the geometry of the electrodes to reach optimum collection efficiency. b) Secondary electrons. c) Heat dissipation on the electrodes.

\subsection{Potentials on the electrode}

In the design of the energy recovery system, the energy distribution of the electron beam was exported from the simulation of the gyro-BWO using MAGIC, as shown in Fig. 25. Table 6 shows the optimum potentials and the collection efficiency when a different number of stages are used. In this calculation, it was assumed that all the electrons were collected on the electrodes without consideration of secondary emissions. The minimum electrode potential was set to be the minimum energy of electrons to avoid backstreaming and the maximum potential was set to be the electron beam voltage which was $40 \mathrm{kV}$ in the gyro-BWO device. It was found that when the number of stages increased beyond four, the collection efficiency did not significantly increase. Four stages were therefore chosen as a compromise between the collection efficiency and complexity of the system.

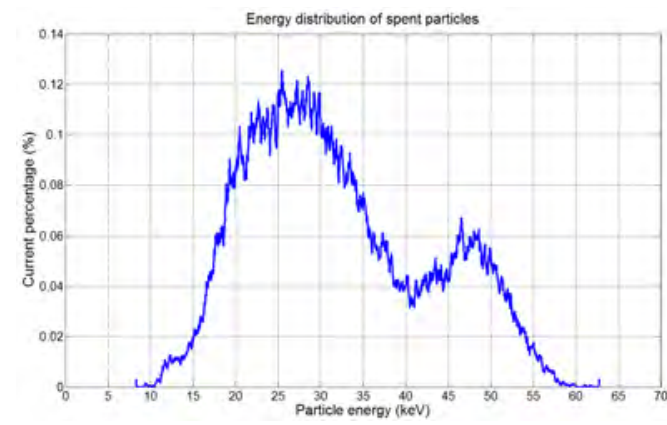

(a) Energy distribution of the spent beam

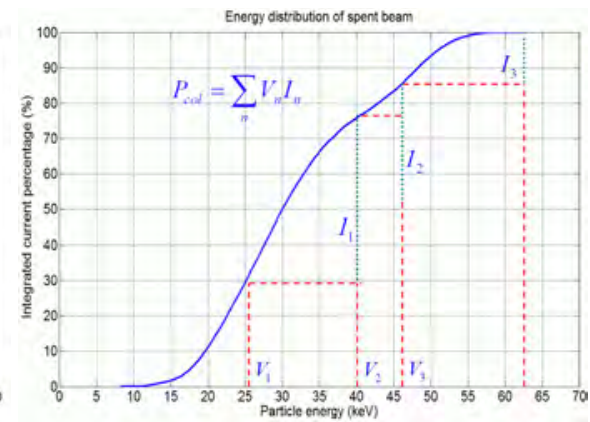

(b) Recovered energy from the spent electrons

Fig. 25. Energy distribution of the spent beam under the condition of $40 \mathrm{kV}$ electron beam voltage, $1.5 \mathrm{~A}$ beam current, beam $\alpha$ of 1.6 , with a cavity magnetic field of $1.75 \mathrm{~T}$.

\subsection{Geometry of the depressed collector and optimization process}

The collection efficiency calculated in section 4.2 assumed that all the spent electrons were sorted by the electric and magnetic field in the collection region. In practice, the distribution of the electric field is determined by the geometry of the electrodes. Proper design of the electrode geometry not only acts to sort the electrons with different kinetic energies, but also to decrease the possibility of secondary emission and to avoid the backstreaming of the electrons in the collector. One way to choose a good geometry is to use a searching algorithm such as a random walk and genetic algorithm to optimize the parameters (Ghosh \& Carter, 2007). 


\begin{tabular}{|c|c|c|c|c|c|c|c|c|}
\hline No. & \multicolumn{7}{|c|}{$\begin{array}{c}\text { Potentials on electrodes (kV) } \\
\text { (relative to ground voltage) }\end{array}$} & $\begin{array}{c}\text { Collection } \\
\text { efficiency }\end{array}$ \\
\hline 1 & -9.24 & - & - & - & - & - & - & $28.8 \%$ \\
\hline 2 & -9.24 & -25.70 & - & - & - & - & - & $63.6 \%$ \\
\hline 3 & -9.24 & -22.14 & -36.57 & - & - & - & - & $75.7 \%$ \\
\hline 4 & -9.24 & -19.55 & -27.31 & -40.00 & - & - & - & $82.5 \%$ \\
\hline 5 & -9.24 & -18.86 & -24.96 & -30.78 & -40.00 & - & - & $85.7 \%$ \\
\hline 6 & -9.24 & -16.98 & -22.14 & -27.22 & -32.66 & -40.00 & - & $87.5 \%$ \\
\hline 7 & -9.24 & -16.82 & -21.33 & -25.47 & -29.53 & -34.23 & -40.00 & $88.7 \%$ \\
\hline
\end{tabular}

Table 6. Collection efficiency for different number of stages.

An optimization program integrating a genetic algorithm was developed to optimize the depressed collector geometry parameters by controlling the MAGIC code without the need to know the source code. The optimization program firstly created an input file by inserting the new set of parameters to the template input file for MAGIC. Then MAGIC was invoked to simulate the new geometry and the result was read by the optimization program to evaluate the parameters. The flow diagram of this process is shown as Fig. 26 (Zhang et al., 2009a).

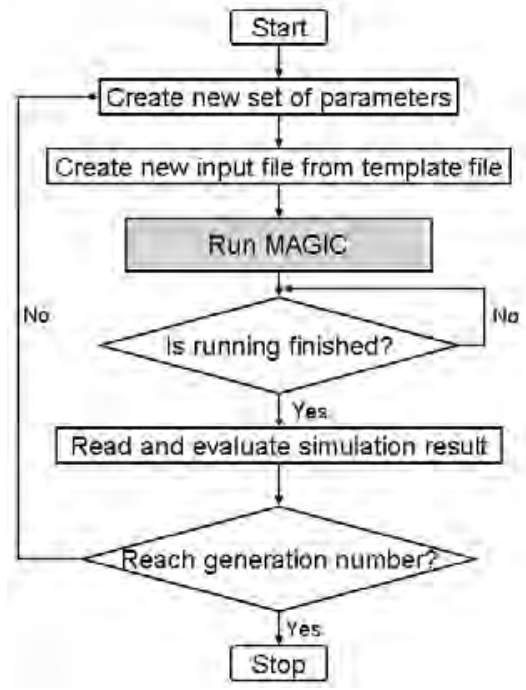

Fig. 26. Flow diagram of the optimization program.

The basic geometry of an electrode is shown in Fig. 27. It is determined completely by 4 parameters, the length of the electrode $(\mathrm{Li})$, the height of the electrode $(\mathrm{Hi})$, the offset from the $\mathrm{Z}$ axis $(\mathrm{O} i)$ and the tilt angle $(A i)$. There should be 16 parameters in a 4-stage collector. However, the outer radius of the depressed collector was restricted to $60 \mathrm{~mm}$, and the overall length was restricted to be $150 \mathrm{~mm}$. Thus 14 parameters were to be optimized. Before the optimization, many simulations were carried out to find a proper range for each parameter to ensure the searching range was as small as possible.

The full geometry of the 4-stage depressed collector is shown in Fig. 28. A gap of $10 \mathrm{~mm}$ between the end of the collection region and the first stage of the collector was left to isolate 


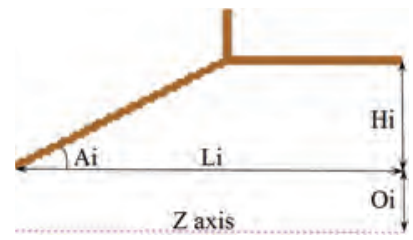

Fig. 27. Geometry of an electrode.

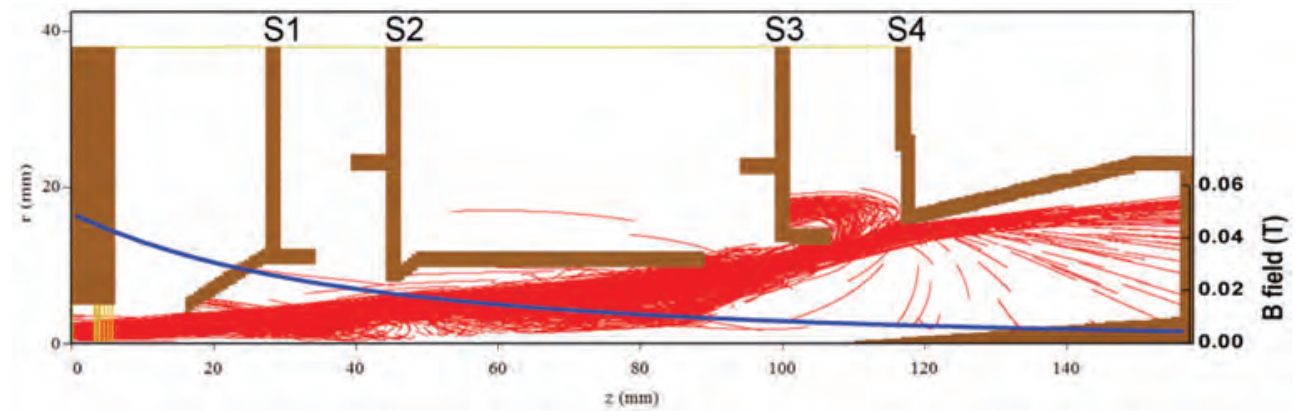

Fig. 28. Full geometry of 4-stage depressed collector.

the high voltage between them. The electrode shapes were modified as shown in S1, S2, S3 and S4 to avoid potential distortion in the simulation when the electric field was applied to the electrodes.

The potentials on each electrode were consistent with Table 6. After each simulation, the average current collected by each collector was read from a MAGIC output data file. Then the collected power was calculated from Eq. 12. The average power of the spent beam was calculated by counting all the energy from the spent electrons. The collection efficiency calculated by the collected power and power of the spent beam was used to evaluate the optimum geometric parameters. The crossover probability, mutation probability, and population size of the genetic algorithm were set to be $0.85,0.05$ and 12 , respectively. The evaluation function is

$$
\eta_{\text {eva }}=\eta_{\text {col }}-W \eta_{\text {back }}
$$

where $\eta_{\text {back }}$ was the percentage of the backstreaming electrons, and $W$ was the weight. In our calculation, $W$ was chosen as 1.5. The optimization was run with the magnetic field of $1.75 \mathrm{~T}$. After 756 iterations, an optimum collection efficiency of $78.7 \%$ was achieved. It was $3.8 \%$ lower than the ideal collection efficiency calculated in the preceding section which assumed all the spent electrons were sorted perfectly. That was because not all the electrons were recovered by the optimum electrode and a small proportion were observed to backstream in the simulation. The trajectories of the spent electrons are also shown in Fig. 28.

By applying the optimum depressed collector with collection efficiency of $78.7 \%$, the overall efficiency of the gyro-BWO was enhanced

$$
\eta_{\text {tot }}=\frac{0.150}{1-0.787 \times(1-0.167)} \times 100 \%=43.6 \%
$$


The overall efficiency was greatly improved by using the energy recovery system. The spent electron beams for different magnetic fields were also simulated and the collection efficiencies were about $78.0 \%-82.0 \%$ under their optimum potentials.

\subsection{Simulation with secondary electron emission}

The secondary electrons have several negative effects on high power microwave devices. First of all, secondary electrons carrying velocities with opposite direction to the primaries will be accelerated by the electrostatic field in the collection region and some of them will backstream. The secondary electrons absorb energy from the electrostatic field and decrease the collection efficiency. Secondly, the backstreaming electrons enter into the RF interaction region, which will generate noise on the microwave output and decrease the performance of the microwave tube. Thirdly, in high average power devices, the backstreaming may contribute an additional thermal power on the thermally stressed waveguide structure (Ling et al., 2000). Thus in depressed collectors, it is essential to reduce the current of secondary electrons to be as low as possible.

Secondary electrons are generally divided into three classes, including the true secondary electrons (TSEs), the rediffused electrons, and the backscattered elastic electrons (Furman \& Pivi, 2002). In our simulation, the rediffused electrons and the backscattered elastic electrons were treated by a uniform model for they had the same physical nature. Generally, the term of "backscattered electrons"(BSEs) was used to indicate these two types of secondary electrons. In MAGIC, the numbers, the energies and the angles of the emitted TSEs were sampled from the probability function of the yield, the energy distribution and the angular distribution by using a Monte Carlo algorithm. Therefore the true secondary yield (SEY), the emitted angular distribution and the emitted-energy spectrum are considered as important quantities in the simulation. Data about the SEY, the emitted angle distribution and the emitted-energy spectrum can be obtained from experiments, and several semiempirical formulas have been developed to fit the experimental data, such as Vaughan's, Furman's and Thomas's equations (Furman \& Pivi, 2002; MAGIC, 2002; Vaughan, 1993).

The scattering process of the BSEs in MAGIC is carried out by ITS (The integrated TIGER Series of Coupled Electron/Photon Monte Carlo Transport Codes) code and it has been proved that the simulation results of the ITS code were in good agreement with the experiments (Halbleib et al., 1992). The "BACKSCATTER"option in MAGIC allows ITS to be invoked automatically to simulate the emission of both the rediffused and backscattered elastic electrons. The TSE and BSE models used in the MAGIC simulations were discussed in detail in ref. (Zhang et al., 2009b).

The optimization simulation was run once again with the secondary electrons considered. Copper was chosen as the material of the electrodes. The collected power taking account of the secondary electrons was revised as

$$
\begin{aligned}
& P_{c o l}=\sum_{n}^{N} V_{n} I_{n}+\sum_{n}^{N} \sum_{j}^{N} \operatorname{SIGN}(n-j) I_{n j}\left(V_{n}-V_{j}\right)-\sum_{n}^{N} V_{n} I_{B n} \\
& \operatorname{SIGN}(n-j)=\left\{\begin{array}{l}
-1, n \leq j \\
1, n>j
\end{array}\right.
\end{aligned}
$$

where $N$ is the number of stages $V_{n}, I_{n}$ and are the potentials and the collected primary current on the $n^{\text {th }}$-stage electrode, respectively. $I_{n j}$ is the current of the secondary electrons emitted 
from the $n^{\text {th }}$-stage electrode and collected on the $j^{t h}$-stage electrode. $I_{B n}$ is the backstreaming current by the secondary electrons emitted from the $n^{\text {th }}$-stage electrode.

In the previous optimum geometry without considering the secondary electrons, a collection efficiency of $78.7 \%$ was obtained in the conditions of beam current of $1.5 \mathrm{~A}$, beam voltage of $40 \mathrm{kV}$, magnetic field of $1.75 \mathrm{~T}$, and the operation frequency of $91.4 \mathrm{GHz}$. When taking account of the secondary electron emission using Vaughan's true secondary emission model, the collection efficiency was reduced from $78.7 \%$ to $69.2 \%$ and the backstreaming increased from $4.5 \%$ to $9.4 \%$. The backstreaming was large thus modifications in the geometry were required and the optimization process was carried out once again. After 552 iterations, an optimum collection efficiency of $69.0 \%$ was achieved when using Vaughan's true secondary emission model, whilst the backstreaming was reduced from $9.4 \%$ to $4.9 \%$. Table 7 presents the predicted collection efficiency and the percentage of the backstreaming current for a range of secondary electron models. From the simulation results, by carefully designing the geometry of the depressed collector, the backstreaming could be reduced to a relatively low level.

\begin{tabular}{|c|c|c|c|}
\hline Cases & $\begin{array}{c}\text { True secondary } \\
\text { model }\end{array}$ & $\begin{array}{c}\text { Collection } \\
\text { efficiency }\end{array}$ & $\begin{array}{c}\text { Percentage of } \\
\text { backstreaming }\end{array}$ \\
\hline without TSEs, without BSEs & - & $75.7 \%$ & $4.79 \%$ \\
\hline \multirow{3}{*}{ with TSEs, without BSEs } & Vaughan & $71.1 \%$ & $4.79 \%$ \\
& Furman & $68.9 \%$ & $4.80 \%$ \\
& Thomas & $73.0 \%$ & $4.80 \%$ \\
\hline without TSEs, with BSEs & - & $73.9 \%$ & $4.89 \%$ \\
\hline \multirow{2}{*}{ with TSEs, with BSEs } & Vaughan & $69.0 \%$ & $4.89 \%$ \\
& Furman & $66.8 \%$ & $4.91 \%$ \\
& Thomas & $71.0 \%$ & $4.90 \%$ \\
\hline
\end{tabular}

Table 7. The collection efficiency and the backstreaming rate in different cases.

From the simulation results, the backstreaming caused by the primary electrons was $4.79 \%$, while TSEs and BSEs only contributed about $0.1 \%$. Between BSEs and TSEs, the backstreaming was mostly composed by BSEs, as the BSEs had a higher energy than the TSEs thus they were better able to overcome the radial electric field and return to the interaction region. Fig. 29 shows the trajectories of the primary electrons, the true secondary electrons and the backscattered electrons in the designed depressed collector when using Vaughan's formula. The reduction of the collection efficiency caused by the three different models of true secondary emission yield did not show a great difference and was about $4 \%$. Each secondary emission model generated a different number of the true secondary electrons and impacted the second and third terms of Eq. 16. The second term was much smaller than the first term since $V_{i}-V_{j}$ was much smaller than $V_{i}$. In the simulation, the potentials on each electrode were $-9.24 \mathrm{kV},-19.55 \mathrm{kV},-27.31 \mathrm{kV},-40.00 \mathrm{kV}$, respectively. From the trajectories of the true secondary electrons in Fig. 29(b), most of the true secondary electrons emitted from the forth, third and second electrodes were collected by the third, second, and first electrode, respectively. That made the second term of Eq. 16 a small value. Since the difference between the collection efficiency and backstreaming rate associated with the different true secondary emission models were found to be small, in subsequent calculations, we only used Vaughan's formula because it has been widely accepted in the literature.

The output frequency of the W-band gyro-BWO can be tuned by adjusting the amplitude of the cavity magnetic field. However the spent beam parameters were also affected by this 


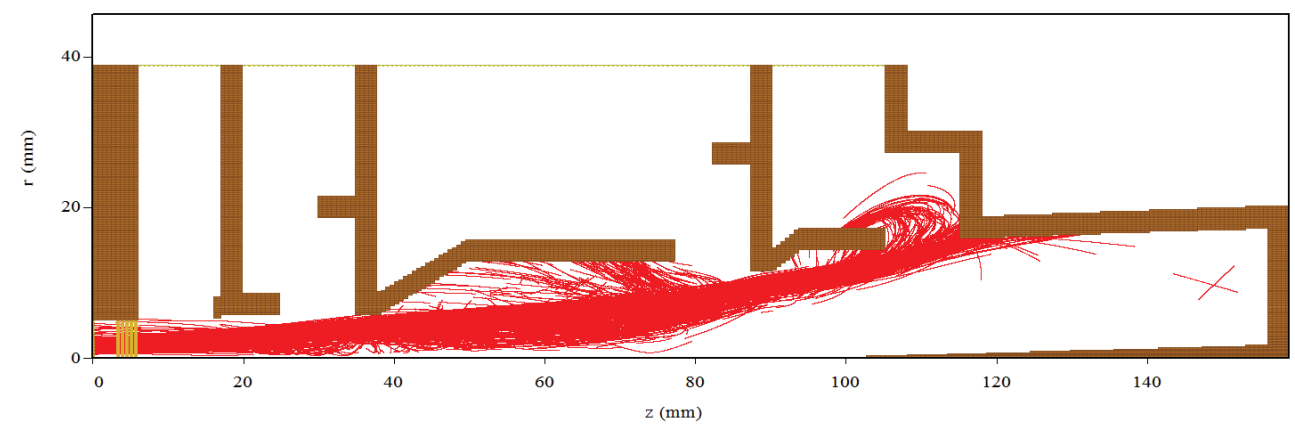

(a) Trajectories of the primary electrons

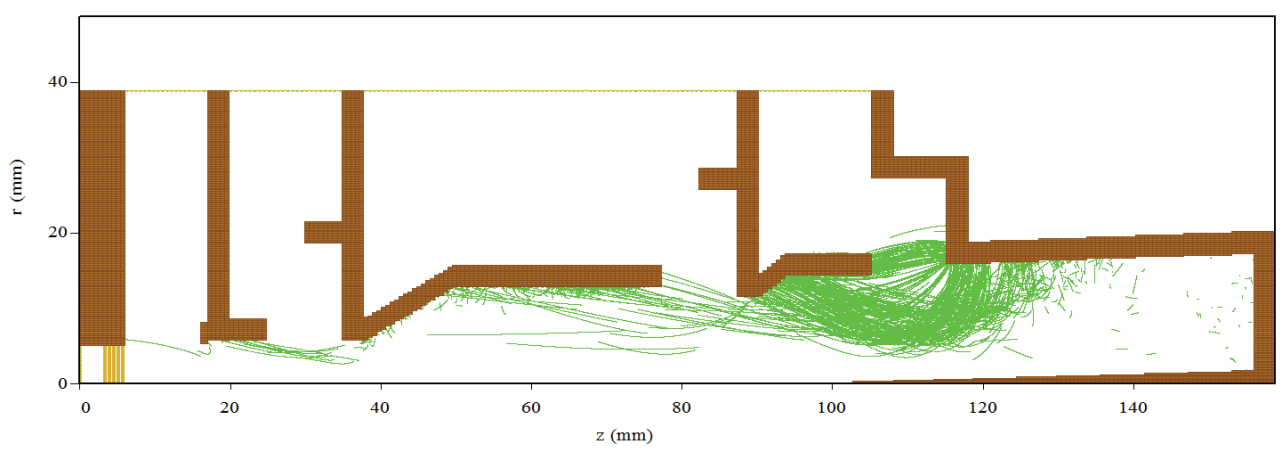

(b) Trajectories of the true secondary electrons

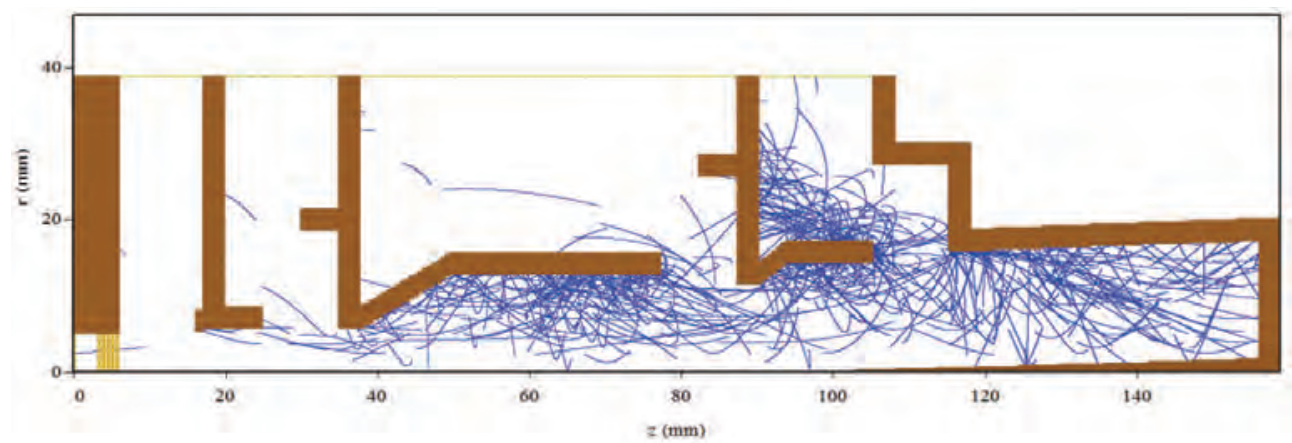

(c) Trajectories of the backscattered electrons

Fig. 29. Trajectories of the electrons in the depressed collector (using Vaughan's formulas). 
tuning. The collection efficiencies and the backstreaming rate of the $\mathrm{W}$-band gyro-BWO were therefore simulated in the whole frequency tuning range for the optimized configuration of the four-stage depressed collector. The collection efficiencies achieved were simulated to be about $70 \%$ and the backstreaming rate was lower than $7 \%$ in the working frequency band.

\subsection{Heat power density distribution on the collector}

To design an effective cooling system for the collector electrodes, the distribution of the heat power dissipated on the surface of the electrodes needs to be evaluated. In MAGIC, there is no way to obtain the heat power on the electrodes directly. However, it provides a command "OBSERVE COLLECTED POWER"to monitor the overall heat dissipation on a conductor. To obtain the heat power distribution on the surface of the electrodes, the four electrodes were divided into a large number of small conductors both in the azimuthal direction and the $z$ direction and the heat power dissipated in each of these conductors was individually recorded, thus an approximate heat power distribution was obtained. The greater the number of conductors, the higher the resolution of the heat power distribution that could be achieved. The heat power densities on the conductors could be calculated by dividing the heat power by the area of the conductors'surface. In the simulation, the maximum heat density was $\sim 240 \mathrm{~W} / \mathrm{cm}^{2}$. It is lower than the thermal stress threshold of the copper material thus the generation of "hot spots" can be avoided.

\section{Conclusion}

In this chapter, the simulations and optimizations of a W-band gyro-BWO including the simulation of a thermionic cusp electron gun which generates an annular, axis-encircling electron beam, the simulation of the beam-wave interaction in the helically corrugated interaction region and the simulation and optimization of an energy recovery system of a 4-stage depressed collector were presented.

The annular-shaped axis-encircling electron beam produced by the cusp electron gun had a beam current of $1.5 \mathrm{~A}$ at an acceleration potential of $40 \mathrm{kV}$, an optimized axial velocity spread $\Delta v_{z} / v_{z}$ of $8 \%$, and a relative $\alpha$ spread $\Delta \alpha / \alpha$ of $10 \%$ at an $\alpha$ value of 1.65 . When driven by such a beam the gyro-BWO was simulated to have a $3 \mathrm{~dB}$ frequency bandwidth of $84-104 \mathrm{GHz}$, output power of $10 \mathrm{~kW}$ with an electronic efficiency of $17 \%$. The optimization of the shape and dimensions of each stage of the depressed collector using a genetic algorithm achieved an overall recovery efficiency of about $70 \%$, with a minimized back-streaming rate of $4.9 \%$. With the addition of a four stage depressed collector an overall efficiency of $40 \%$ was simulated for the gyro-BWO.

\section{References}

Arnone, D. D., Ciesla, C. M., Corchia, A., Egusa, S., Pepper, M., Chamberlain, J. M., Bezant, C., Linfield, E. H., Clothier, R. \& Khammo, N. (1999). Applications of terahertz (THz) technology to medical imaging, in J. M. Chamberlain (ed.), Society of Photo-Optical Instrumentation Engineers (SPIE) Conference Series, Vol. 3828, pp. 209-219.

Bratman, V. L., Denisov, G. G., Samsonov, S. V, Cross, A. W., Phelps, A. D. R. \& He, W. (2007). High-efficiency wideband gyro-TWTs and gyro-BWOs with helically corrugated waveguides, Radiophys. and Quantum Elect. 50: 95-107. 
Bratman, V. L., Denisov, G. G., Manuilov, V. N., Samsonov, S. V. \& Volkov, A. B. (2001). Development of helical-waveguide gyro-devices based on low-energy electron beams, Digest of Int. Conf. Infrared and Millimeter Waves, Toulouse, France pp. 5-105.

Bratman, V. L., Cross, A. W., Denisov, G. G., He, W., Phelps, A. D. R., Ronald, K., Samsonov, S. V., Whyte, C. G. \& Young, A. R. (2000). High-gain wide-band gyrotron traveling wave amplifier with a helically corrugated waveguide, Phys. Rev. Lett. 84(12): 2746-2749.

Burt, G., Samsonov, S. V., Phelps, A. D. R., Bratman, V. L., Ronald, K., Denisov, G. G., He, W., Young, A., Cross, A. W. \& Konoplev, I. V. (2005). Microwave pulse compression using a helically corrugated waveguide, IEEE Trans. Plasma Sci. 33(2): 661-667.

Burt, G., Samsonov, S. V., Ronald, K., Denisov, G. G., Young, A. R., Bratman, V. L., Phelps, A. D. R., Cross, A. W., Konoplev, I. V., He, W., Thomson, J. \& Whyte, C. G. (2004). Dispersion of helically corrugated waveguides: Analytical, numerical, and experimental study, Phys. Rev. E 70(4): 046402.

Chen, F. F. (1974). Introduction to Plasma Physics, Plenum Press, New York.

Chu, K. R. (1978). Theory of electron cyclotron maser interaction in a cavity at the harmonic frequencies, Phys. Fluids 21(12): 2354-2364.

Cooke, S. J., Cross, A. W., He, W. \& Phelps, A. D. R. (1996). Experimental operation of a cyclotron autoresonance maser oscillator at the second harmonic, Phys. Rev. Lett. 77(23): 4836-4839.

Cross, A. W., He, W., Phelps, A. D. R., Ronald, K., Whyte, C. G., Young, A. R., Robertson, C. W., Rafferty, E. G. \& Thomson, J. (2007). Helically corrugated waveguide gyrotron traveling wave amplifier using a thermonic cathode electron gun, Appl. Phys. Lett. 90: 253501.

Denisov, G. G., Bratman, V. L., Cross, A. W., He, W., Phelps, A. D. R., Ronald, K., Samsonov, S. V. \& Whyte, C. G. (1998). Gyrotron traveling wave amplifier with a helical interaction waveguide, Phys. Rev. Lett. 81(25): 5680-5683.

Denisov, G. G., Bratman, V. L., Phelps, A. D. R. \& Samsonov, S. V. (1998). Gyro-TWT with a helical operating waveguide: New possibilites to enhance efficiency and frequency bandwidth, IEEE Trans. Plasma Sci. 26(3): 508-518.

Destler, W. W. \& Rhee, M. J. (1977). Radial and axial compression of a hollow electron beam using an asymmetric magnetic cusp, Phys. Fluids 20(9): 1582-1584.

Donaldson, C. R., He, W., Cross, A. W., Li, F., Phelps, A. D. R., Zhang, L., Ronald, K., Robertson, C. W., Whyte, C. G. \& Young, A. R. (2010). A cusp electron gun for millimeter wave gyrodevices, Appl. Phys. Lett. 96(14): 141501.

Donaldson, C. R., He, W., Cross, A. W., Phelps, A. D. R., Li, F., Ronald, K., Robertson, C. W., Whyte, C. G., Young, A. R. \& Zhang, L. (2009). Design and numerical optimization of a cusp-gun-based electron beam for millimeter-wave gyro-devices, IEEE Trans. Plasma Sci. 37(11): 2153-2157.

Furman, M. A. \& Pivi, M. T. (2002). Probabilistic model for the simulation of secondary electron emission, Phys. Rev. Spec. Top., Accel. Beams 5(12): 124404.

Gallagher, D. A., Barsanti, M., Scafuri, F. \& Armstrong, C. (2000). High-power cusp gun for harmonic gyro-device applications, IEEE Trans. Plasma Sci. 28(3): 695-699.

Ganguly, A. K. \& Ahn, S. (1989). Non-linear analysis of the gyro-BWO in three dimensions, Int. J. Electronics 67(2): 261-276. 
Ghosh, T. K. \& Carter, R. G. (2007). Optimization of multistage depressed collectors, IEEE Trans. Electron Devices 54(8): 2031-2039.

Goplen, B., Ludeking, L., Smithe, D. \& Warren, G. (1995). User-configurable MAGIC for electromagnetic PIC calculations, Comput. Phys, Commun, 87: 54-86.

Halbleib, J. A., Kensek, R. P., Valdez, G. D., Mehlhorn, T. A., Seltzer, S. M. \& Berger, M. J. (1992). ITS: The integrated TIGER series of electron/photon transport codes - Version 3.0, IEEE Trans. Nucl. Sci. 39(4): 1025-1030.

He, W., Cooke, S. J., Cross, A. W. \& Phelps, A. D. R. (2001). Simultaneous axial and rotational electron beam velocity measurement using a phosphor scintillator, Rev. Sci. Inst. 72(5): 2268-2270.

He, W., Ronald, K., Young, A. R., Cross, A. W., Phelps, A. D. R., Whyte, C. G., Rafferty, E. G., Thomson, J., Robertson, C. W., Speirs, D. C., Samsonov, S. V., Bratman, V. L. \& Denisov, G. G. (2005). Gyro-BWO experiments using a helical interaction waveguide, IEEE Trans. Electron Devices 52(5): 839 - 844.

He, W., Whyte, C. G., Rafferty, E. G., Cross, A. W., Phelps, A. D. R., Ronald, K., Young, A. R., Robertson, C. W., Speirs, D. C. \& Rowlands, D. H. (2008). Axis-encircling electron beam generation using a smooth magnetic cusp for gyrodevices, Appl. Phys. Lett. 93: 121501.

He, W., Donaldson, C. R., Li, F., Zhang, L., Cross, A. W., Phelps, A. D. R., Ronald, K., Robertson, C. W., Whyte, C. G. \& Young, A. R. (2011). W-band gyro-devices using helically corrugated waveguide and cusp gun: design, simulation and experiment, TST 4(1): 9 - 19.

Idehara, T., Ogawa, I., Mitsudo, S., Iwata, Y., Watanabe, S., Itakura, Y., Ohashi, K., Kobayashi, H., Yokoyama, T., Zapevalov, V. E., Glyavin, M. Y., Kuftin, A. N., Malgin, O. V. \& Sabchevski, S. P. (2004). A high harmonic gyrotron with an axis-encircling electron beam and a permanent magnet, IEEE Trans. Plasma Sci. 32(3): 903-909.

Imai, T., Kobayashi, N., Temkin, R., Thumm, M., Tran, M. Q. \& Alikaev, V. (2001). Iter R \& D: Auxiliary systems: Electron cyclotron heating and current drive system, Fusion Eng. Des. 55(2-3): 281 - 289.

Jeon, S. G., Baik, C. W., Baik, D. H., Kim, D. H., Park, G. S., Sato, N. \& Yokoo, K. (2002). Study on velocity spread for axis-encircling electron beams generated by single magnetic cusp, Appl. Phys. Lett. 80: 3703.

Kou, C. S., Chen, S. H., Barnett, L. R., Chen, H. Y. \& Chu, K. R. (1993). Experimental study of an injection-locked gyrotron backward-wave oscillator, Phys. Rev. Lett. 70(7): 924-927.

Li, F., He, W., Cross, A. W., Donaldson, C. R., Zhang, L., Phelps, A. D. R. \& Ronald, K. (2010). Design and simulation of a $\sim 390 \mathrm{GHz}$ seventh harmonic gyrotron using a large orbit electron beam, J. Phys. D: Appl. Phys. 43(15): 155204.

Ling, G., Piosczyk, B. \& Thumm, M. (2000). A new approach for a multistage depressed collector for gyrotrons, IEEE Trans. Plasma Sci. 28(3): 606-613.

Ludeking, L., Smithe, D. \& Gray, T. (2003). Introduction to MAGIC, Mission Research Corporation.

MAGIC (2002). MAGIC User's Manual, Mission Research Corporation.

Manheimer, W. M., Mesyats, G. \& Petelin, M. I. (1994). Applications of High-power Microwaves, Artech House.

McDermott, D. B., Balkcum, A. J. \& Luhmann Jr., N. C. (1996). 35-GHz 25-kW CW low-voltage third-harmonic gyrotron, IEEE Trans. Plasma Sci. 24(3): 613-629. 
McStravick, M., Samsonov, S. V., Ronald, K., Mishakin, S. V., He, W., Denisov, G. G., Whyte, C. G., Bratman, V. L., Cross, A. W., Young, A. R., MacInnes, P., Robertson, C. W. \& Phelps, A. D. R. (2010). Experimental results on microwave pulse compression using helically corrugated waveguide, J. Appl. Phys. 108(5): 054908-054908-4.

Neugebauer, W. \& Mihran, T. G. (1972). A ten-stage electrostatic depressed collector for improving klystron efficiency, IEEE Trans. Electron Devices 19(1): 111-121.

Nguyen, K. T., Smithe, D. N. \& Ludeking, L. D. (1992). The double-cusp gyro-gun, IEDM Tech. Dig. pp. 219-222.

Nusinovich, G. S. \& Dumbrajs, O. (1996). Theory of gyro-backward wave oscillators with tapered magnetic field and waveguide cross section, IEEE Trans. Plasma Sci. 24(3): 620-629.

Park, S. Y., Kyser, R. H., Armstrong, C. M., Parker, R. K. \& Granatstein, V. L. (1990). Experimental study of a Ka-band gyrotron backward-wave oscillator, IEEE Trans. Plasma Sci. 18(3): 321 -325.

Pierce, J. R. (1954). Theory and Design of Electron Beams, Van Nostrand.

Rhee, M. J. \& Destler, W. W. (1974). Relativistic electron dynamics in a cusped magnetic field, Phys. Fluids 17(8): 1574-1581.

Samsonov, S. V., Phelps, A. D. R., Bratman, V. L., Burt, G., Denisov, G. G., Cross, A. W., Ronald, K., He, W. \& Yin, H. (2004). Compression of frequency-modulated pulses using helically corrugated waveguides and its potential for generating multigigawatt $\mathrm{rf}$ radiation, Phys. Rev. Lett. 92(11): 118301.

Scheitrum, G. P., Symons, R. S. \& True, R. B. (1989). Low velocity spread axis-encircling electron beams forming system, IEDM Tech. Dig. pp. 743-746.

Scheitrum, G. P. \& True, R. (1981). A triple pole piece magnetic field reversal element for generation of high rotational energy beam, IEDM Tech. Dig. 27: 332-335.

Schmidt, G. (1962). Nonadiabatic particle motion in axialsymmetric fields, Phys. Fluids 5(8): 994-1002.

Sinnis, J. \& Schmidt, G. (1963). Experiment trajectory analysis of charged particles in a cusped geometry, Phys. Fluids 6(6): 841-845.

Smirnova, T. I., Smirnov, A. I., Clarkson, R. B. \& Belford, R. L. (1995). W-Band (95 GHz) EPR spectroscopy of nitroxide radicals with complex proton hyperfine structure: Fast motion, J. Phys. Chem. 99(22): 9008-9016.

Sprangle, P. \& Smith, R. A. (1980). The nonlinear theory of efficiency enhancement in the electron cyclotron maser (gyrotron), J. Appl. Phys. 51(6): 3001-3007.

Sterzer, F. \& Princeton, N. J. (1958). Improvement of traveling-wave tube efficiency through collector potential depression, IRE Trans. Electron Devices 5(4): 300-305.

Vaughan, M. (1993). Secondary emission formulas, IEEE Trans. Electron Devices 40(4): 830.

Walter, M. T., Gilgenbach, R. M., Luginsland, J. W., Hochman, J. M., Rintamaki, J. I., Jaynes, R. L., Lau, Y. Y. \& Spencer, T. A. (1996). Effects of tapering on gyrotron backward-wave oscillators, IEEE Trans. Plasma Sci. 24(3): 636-647.

Wang, Q. S., Huey, H. E., McDermott, D. B., Hirata, Y. \& Luhmann Jr., N. C. (2000). Design of a W-band second-harmonic $\mathrm{TE}_{02}$ gyro-TWT amplifier, IEEE Trans. Plasma Sci. 28(6): 2232-2237.

Wang, Q. S., McDermott, D. B., Chong, C. K., Kou, C. S., Chu, K. R. \& C., L. J. N. (1994). Stable 1 MW, third-harmonic gyro-TWT amplifier, IEEE Trans. Plasma Sci. 22(5): 608-615. 
Wilson, J. D., Wintucky, E. G., Vaden, K. R., Force, D. A., Krainsky, I. L., Simons, R. N., Robbins, N. R., Menninger, W. L., Dibb, D. R. \& Lewis, D. E. (2007). Advances in space traveling-wave tubes for NASA missions, Proc. IEEE 95(10): 1958-1967.

Zhang, L., He, W., Cross, A. W., Phelps, A. D. R., Ronald, K. \& Whyte, C. G. (2009a). Design of an energy recovery system for a gyrotron backward-wave oscillator, IEEE Trans. Plasma Sci. 37(3): 390-394.

Zhang, L., He, W., Cross, A. W., Phelps, A. D. R., Ronald, K. \& Whyte, C. G. (2009b). Numerical Optimization of a Multistage Depressed Collector With Secondary Electron Emission for an X-band Gyro-BWO, IEEE Trans. Plasma Sci. 37(12): 2328 - 2334. 